\title{
Complex Dynamics of a Discrete-Time Predator-Prey System with Ivlev Functional Response
}

\author{
Hunki Baek (iD) \\ Department of Mathematics Education, Catholic University of Daegu, Gyeongsan, Gyeongbuk 712-702, Republic of Korea \\ Correspondence should be addressed to Hunki Baek; hkbaek@cu.ac.kr
}

Received 27 July 2017; Revised 25 November 2017; Accepted 19 December 2017; Published 8 February 2018

Academic Editor: David Bigaud

Copyright (c) 2018 Hunki Baek. This is an open access article distributed under the Creative Commons Attribution License, which permits unrestricted use, distribution, and reproduction in any medium, provided the original work is properly cited.

\begin{abstract}
The dynamics of a discrete-time predator-prey system with Ivlev functional response is investigated in this paper. The conditions of existence for flip bifurcation and Hopf bifurcation in the interior of $\mathbb{R}_{+}^{2}$ are derived by using the center manifold theorem and bifurcation theory. Numerical simulations are presented not only to substantiate our theoretical results but also to illustrate the complex dynamical behaviors of the system such as attracting invariant circles, periodic-doubling bifurcation leading to chaos, and periodic-halving phenomena. In addition, the maximum Lyapunov exponents are numerically calculated to confirm the dynamical complexity of the system. Finally, we compare the system to discrete systems with Holling-type functional response with respect to dynamical behaviors.
\end{abstract}

\section{Introduction}

In population dynamics, continuous-time dynamical systems, such as a Lotka-Volterra system, have been used to understand the interaction between ecological species [18]. In recent years, discrete-time population systems also come to the fore due to the following reasons [9-16]: Firstly, discrete-time systems are more suitable than continuoustime systems to describe populations with nonoverlapping generations. Secondly, they can produce more complex and rich dynamical behaviors than continuous-time systems. Finally, we can get more accurate numerical simulation results from discrete-time systems compared with continuous-time systems.

In order to investigate the dynamical relationship between two species, predator and prey, one of important components we should pay attention to is the predator's rate of feeding on the prey, which is called functional response. In this context, Holling [17] suggested three different kinds of functional response for different kinds of species to model the phenomena of predation, which are called Holling's type I, II, and III. These functional responses are not only monotonically increasing, but also uniformly bounded functions in the first quadratic. The authors $[18,19]$ introduced another Holling-type functional response called Holling-type IV functional response or Monod-Haldane functional response. Ivlev [20] also suggested a well-known functional response, called Ivlev functional response, in the following form;

$$
p(x)=h(1-\exp (-c x)),
$$

where $h, c$ are positive constants and represent the maximum rate of predation and the decrease in motivation to hunt, respectively.

There are many works to investigate predator-prey system with Ivlev-type functional response. The results indicated that predator-prey systems with Ivlev-type have wide applicabilities in ecology, such as dynamics in predator-prey system [21$25]$, host-parasitoid system $[26,27]$, and animal coat pattern [28].

Taking into account this functional response, we will consider the following predator-prey system with Ivlev functional response:

$$
\begin{aligned}
& \frac{d x}{d t}=a x\left(1-\frac{x}{b}\right)-h(1-\exp (-c x)) y, \\
& \frac{d y}{d t}=-D y+k(1-\exp (-c x)) y,
\end{aligned}
$$

where $x(t)$ and $y(t)$ are functions of time representing the population densities of the prey and the predator, respectively. 
All constants are positive. The parameter $a$ means the growth rate of the prey and $b$ is the carrying capacity. The constant $D$ denotes the death rate of the predator, and $k$ is the rate of conversion of a consumed prey to a predator.

Now, we will apply the forward Euler scheme to system (2) to obtain the following discrete-time predator-prey system with Ivlev functional response:

$$
\begin{aligned}
& x \longrightarrow x+\delta\left(a x\left(1-\frac{x}{b}\right)-h(1-\exp (-c x)) y\right), \\
& y \longrightarrow y+\delta(-D y+k(1-\exp (-c x)) y),
\end{aligned}
$$

where $\delta>0$ is the time step size.

Until now, many researchers have concerned themselves with discrete-time predator-prey systems with Holling-type functional response $([9,12-16,29,30])$. Most of these works have dealt with the existence and stability of fixed points and bifurcation phenomena such as flip bifurcation, saddlenode bifurcation, or Hopf bifurcation. On the contrary, in this paper, we will take into account a discrete predator-prey system with Ivlev functional response which has not been studied well until now.

The purpose of this paper is to analyze dynamical behaviors of system (3) and to observe a flip bifurcation and Hopf bifurcation by using the center manifold theorem and bifurcation theory. Moreover, numerical simulations are presented not only to substantiate our theoretical results but also to illustrate the complex dynamics of system (3) which are difficult to explain theoretically.

This paper is organized as follows. In Section 2, we investigate the existence and local stability of the fixed points of system (3). In Section 3, we show that there exist some values of parameters such that system (3) undergoes a flip bifurcation and Hopf bifurcation. In Section 4, we display the numerical simulation to provide numerical evidence for theoretical results and to exhibit numerically various dynamical behaviors including periodic doubling and chaotic phenomena. In addition, we calculate the maximum Lyapunov exponents to confirm the dynamical complexity of system (3). In Section 5, we summarize the results of this paper and compare system (3) to the discrete systems with Holling-type functional response with respect to dynamical behaviors.

\section{The Existence and Stability of the Fixed Points}

In this section, from the point of view of biology, we will focus on the dynamical behaviors of system (3) in the closed first quadrant $\mathbb{R}_{+}^{2}$. In order to determine the fixed points of system (3) we have to solve the nonlinear system given by

$$
\begin{aligned}
& x=x+\delta\left(a x\left(1-\frac{x}{b}\right)-h(1-\exp (-c x)) y\right), \\
& y=y+\delta(-D y+k(1-\exp (-c x)) y) .
\end{aligned}
$$

Simple calculation yields that there are at most three fixed points:

(i) $E_{0}(0,0)$ is the origin;

(ii) $E_{1}(b, 0)$ is the axial fixed point in the absence of the predator;

(iii) $E_{2}\left(x^{*}, y^{*}\right)$ is the interior fixed point, where

$$
\begin{aligned}
& x^{*}=\frac{1}{c} \log \left(\frac{k}{k-D}\right) \\
& y^{*}=\frac{a k}{h c D}\left(1-\frac{1}{b c} \log \left(\frac{k}{k-D}\right)\right) \log \left(\frac{k}{k-D}\right)
\end{aligned}
$$

exists if and only if the following condition is satisfied:

$$
D<k(1-\exp (-b c)) .
$$

We will study the stability of these fixed points by computing the variation matrix corresponding to each fixed point. The Jacobian matrix of system (3) at the state variable $(x, y)$ is given by

$$
J(x, y)=\left(\begin{array}{cc}
1+a \delta-\frac{2 a \delta x}{b}-c h \delta y \exp (-c x) & h \delta(\exp (-c x)-1) \\
c k \delta y \exp (-c x) & 1-D \delta+k \delta(1-\exp (-c x))
\end{array}\right)
$$

The characteristic equation of the Jacobian matrix $J(x, y)$ can be obtained as

$$
\lambda^{2}-p(x, y) \lambda+q(x, y)=0,
$$

where $p(x, y)$ is the trace and $q(x, y)$ is the determinant of the Jacobian matrix $J(x, y)$ which are defined as

$$
\begin{aligned}
& p(x, y)=2+\delta\left(a-D+k-\frac{2 a x}{b}\right)-\delta(c h y+k) \\
& \cdot \exp (-c x) \\
& q(x, y)=\operatorname{chk} \delta^{2} y(1-\exp (-c x)) \exp (-c x)
\end{aligned}
$$

$$
\begin{aligned}
& +(1-D \delta+k \delta(1-\exp (-c x))) \\
& \cdot\left(1+\frac{a \delta(b-2 x)}{b}-c h \delta y \exp (-c x)\right) .
\end{aligned}
$$

In order to determine the stability of the fixed points of system (3), we give the following lemma which can be easily proved by the relations between roots and coefficients of the quadratic equation $(8)[9,30]$.

Lemma 1. Let $F(\lambda)=\lambda^{2}-B \lambda+C$. Suppose that $F(1)>0$ and $\lambda_{1}$ and $\lambda_{2}$ are the two roots of $F(\lambda)=0$. Then 
(1) $\left|\lambda_{1}\right|<1$ and $\left|\lambda_{2}\right|<1$ if and only if $F(-1)>0$ and $C<1$;

(2) $\left|\lambda_{1}\right|<1$ and $\left|\lambda_{2}\right|>1$ (or $\left|\lambda_{1}\right|>1$ and $\left|\lambda_{2}\right|<1$ ) if and only if $F(-1)<0$;

(3) $\left|\lambda_{1}\right|>1$ and $\left|\lambda_{2}\right|>1$ if and only if $F(-1)>0$ and $C>1$

(4) $\lambda_{1}=-1$ and $\left|\lambda_{2}\right| \neq 1$ if and only if $F(-1)=0$ and $B \neq-2,0$;

(5) $\lambda_{1}$ and $\lambda_{2}$ are complex and $\left|\lambda_{1}\right|=\left|\lambda_{2}\right|=1$ if and only if $B^{2}-4 C<0$ and $C=1$.

Let $\lambda_{1}$ and $\lambda_{2}$ be the two roots of (8), which are called eigenvalues of the fixed point $(x, y)$. We recall some definitions of topological types for a fixed point $(x, y)[12$, 14, 32]. A fixed point $(x, y)$ is called a sink if $\left|\lambda_{1}\right|<1$ and $\left|\lambda_{2}\right|<1$, so the sink is locally asymptotically stable. $(x, y)$ is called a source if $\left|\lambda_{1}\right|>1$ and $\left|\lambda_{2}\right|>1$, so the source is locally unstable. $(x, y)$ is called a saddle if $\left|\lambda_{1}\right|<1$ and $\left|\lambda_{2}\right|>1$ (or $\left|\lambda_{1}\right|>1$ and $\left.\left|\lambda_{2}\right|<1\right)$. And $(x, y)$ is called nonhyperbolic if either $\left|\lambda_{1}\right|=1$ or $\left|\lambda_{2}\right|=1$.

Theorem 2. For the fixed point $E_{0}(0,0)$, one has the following topological types:

(1) $E_{0}$ is a source if $\delta>2 / D$;

(2) $E_{0}$ is a saddle if $0<\delta<2 / D$;

(3) $E_{0}$ is nonhyperbolic if $\delta=2 / D$.

Proof. It is easy to get that the Jacobian matrix $J$ at $E_{0}$ is given by

$$
J\left(E_{0}\right)=\left(\begin{array}{cc}
1+a \delta & 0 \\
0 & 1-D \delta
\end{array}\right)
$$

Hence the eigenvalues of the matrix are $1+a \delta$ and $1-D \delta$. Therefore, it is easy to show that (1)-(3) hold.

Theorem 3. For the fixed point $E_{1}(b, 0)$, one has the following topological types:

(1) $D>k(1-\exp (-b c))$ :

(i) $E_{1}$ is $a \operatorname{sink}$ if $\delta<\min \{2 / a, 2 /(D+k(\exp (-b c)-$ 1))\},

(ii) $E_{1}$ is a source if $\delta>\max \{2 / a, 2 /(D+$ $k(\exp (-b c)-1))\}$,

(iii) $E_{1}$ is nonhyperbolic if either $\delta=2 / a$ or $\delta=$ $2 /(D+k(\exp (-b c)-1))$

(2) $D<k(1-\exp (-b c))$ :

(i) $E_{1}$ is a saddle if $\delta<2 / a$,

(ii) $E_{1}$ is a source if $\delta>2 / a$,

(iii) $E_{1}$ is nonhyperbolic if $\delta=2 / a$.

(3) $D=k(1-\exp (-b c)): E_{1}$ is always nonhyperbolic.
Proof. The Jacobian matrix $J$ at $E_{1}$ can be obtained as

$$
J\left(E_{1}\right)=\left(\begin{array}{cc}
1-a \delta & h \delta(\exp (-b c)-1) \\
0 & 1-D \delta+k \delta(1-\exp (-b c))
\end{array}\right) .
$$

Then we have the eigenvalues of the characteristic equation (8) as follows:

$$
\begin{aligned}
& \lambda_{1}=1-a \delta \\
& \lambda_{2}=1-D \delta+k \delta(1-\exp (-b c)) .
\end{aligned}
$$

Therefore elementary calculation yields that the theorem holds.

It follows from Theorem 3 that for the fixed point $E_{1}(b, 0)$, if $(a, b, c, D, k, \delta) \in F_{1}$, where

$$
\begin{aligned}
F_{1} & =\left\{(a, b, c, D, k, \delta): \delta=\frac{2}{a}, D\right. \\
& \neq k(1-\exp (-b c)), \delta \neq \frac{2}{D+k(\exp (-b c)-1)}, b \\
& >0, c>0, D>0, k>0, \delta>0\},
\end{aligned}
$$

then one of the eigenvalues of the fixed point $E_{1}(b, 0)$ is -1 and the other is neither -1 nor 1 . Thus system (3) can undergo a flip bifurcation due to the stability of the fixed point $E_{1}(b, 0)$ when parameters vary in the small neighborhood of $F_{1}$. In fact, if the parameters of system (3) are in $F_{1}$, then a center manifold of system (3) at $E_{1}(b, 0)$ is $y=0$, and if system (3) restricted to this center manifold is the same as the logistic system, $d x / d t=a x(1-x / b)$. Thus in this case the predator becomes extinction; on the other hand, the prey undergoes the period-doubling bifurcation to chaos in the sense of LiYorke for suitable bifurcation parameter $\delta$.

Theorem 4. If $D<k(1-\exp (-b c))$ and $(b c-\log (k /(k-$ $D)))(D-(k-D) \log (k /(k-D)))<D \log (k /(k-D))$ are satisfied, then system (3) has a unique positive fixed point $E_{2}\left(x^{*}, y^{*}\right)$. Moreover, one has the following topological types for the positive fixed point $E_{2}$ :

(1) $E_{2}$ is a sink if one of the following conditions is satisfied:

$$
\begin{aligned}
& \text { (i) }-2 \sqrt{\Delta_{1}} \leq \Delta_{2} \text { and } \delta<-\Delta_{2} / \Delta_{1} \text {, } \\
& \text { (ii) } \Delta_{2}<-2 \sqrt{\Delta_{1}} \text { and } \delta<\left(-\Delta_{2}-\sqrt{\Delta_{2}^{2}-4 \Delta_{1}}\right) / \Delta_{1} \text {. }
\end{aligned}
$$

(2) $E_{2}$ is a source if one of the following conditions is satisfied:

$$
\begin{aligned}
& \text { (i) }-2 \sqrt{\Delta_{1}} \leq \Delta_{2} \text { and } \delta>-\Delta_{2} / \Delta_{1} \text {, } \\
& \text { (ii) } \Delta_{2}<-2 \sqrt{\Delta_{1}} \text { and } \delta>\left(-\Delta_{2}+\sqrt{\Delta_{2}^{2}-4 \Delta_{1}}\right) / \Delta_{1} \text {. }
\end{aligned}
$$

(3) $E_{2}$ is a saddle if $\Delta_{2}<-2 \sqrt{\Delta_{1}}$ and $\left(-\Delta_{2}-\right.$ $\left.\sqrt{\Delta_{2}^{2}-4 \Delta_{1}}\right) / \Delta_{1}<\delta<\left(-\Delta_{2}+\sqrt{\Delta_{2}^{2}-4 \Delta_{1}}\right) / \Delta_{1}$. 
(4) $E_{2}$ is nonhyperbolic if one of the following conditions is satisfied:

$$
\begin{aligned}
& \text { (i) }-2 \sqrt{\Delta_{1}}<\Delta_{2} \text { and } \delta=-\Delta_{2} / \Delta_{1} \text {, } \\
& \text { (ii) } \Delta_{2}<-2 \sqrt{\Delta_{1}} \text { and } \delta=\left(-\Delta_{2} \pm \sqrt{\Delta_{2}^{2}-4 \Delta_{1}}\right) / \Delta_{1} \text {. }
\end{aligned}
$$

Here $\Delta_{1}=(a(k-D) / b c)(b c-\log (k /(k-D))) \log (k /(k-D))$ and $\Delta_{2}=(a / b c D)\left((k-D)(\log (k /(k-D)))^{2}+((b c-2) D-\right.$ $b c k) \log (k /(k-D))+b c D)$.

Proof. Let $\Delta_{1}=(a(k-D) / b c)(b c-\log (k /(k-D))) \log (k /(k-$ $D))$ and $\Delta_{2}=(a / b c D)\left((k-D)(\log (k /(k-D)))^{2}+((b c-2) D-\right.$ $b c k) \log (k /(k-D))+b c D)$. Since $D<k(1-\exp (-b c))$, we have $\log (k /(k-D))<b c$, which implies that $\Delta_{1}>0$. Also, simple calculations yield that $\Delta_{2}=(a / b c D)((b c-\log (k /(k-D)))(D-$ $(k-D) \log (k /(k-D)))-D \log (k /(k-D)))$. Thus $\Delta_{2}<0$ by the hypotheses. From elementary calculation, we obtain that $p\left(x^{*}, y^{*}\right)=\Delta_{2} \delta+2$ and $q\left(x^{*}, y^{*}\right)=\Delta_{1} \delta^{2}+\Delta_{2} \delta+1$ in (8). Now consider $F(\lambda)=\lambda^{2}-B \lambda+C$ as in Lemma 1 , where $B=\Delta_{2} \delta+2$ and $C=\Delta_{1} \delta^{2}+\Delta_{2} \delta+1$. Then we can easily see that

\section{$F(1)$}

$$
\begin{aligned}
& \quad=\frac{a(k-D) \delta^{2}}{b c}\left(b c-\log \left(\frac{k}{k-D}\right)\right) \log \left(\frac{k}{k-D}\right) \\
& =\Delta_{1} \delta^{2}>0, \\
& F(-1)=\Delta_{1} \delta^{2}+2 \Delta_{2} \delta+4 .
\end{aligned}
$$

It follows form Lemma 1 that one of roots of $F(\lambda)=0$ is -1 and the other is neither -1 nor 1 if and only if $\Delta_{2}<-2 \sqrt{\Delta_{1}}$ and $\delta=\left(-\Delta_{2} \pm \sqrt{\Delta_{2}^{2}-4 \Delta_{1}}\right) / \Delta_{1}$, and the roots of $F(\lambda)=0$ are a pair of conjugate complex numbers with modulus 1 if and only if $-2 \sqrt{\Delta_{1}}<\Delta_{2}$ and $\delta=-\Delta_{2} / \Delta_{1}$. Thus, it is easy to see that the theorem holds.

Now consider the following sets;

$$
\begin{aligned}
F_{2} & =\left\{(a, b, c, D, k, \delta) \mid \delta=\frac{-\Delta_{2}-\sqrt{\Delta_{2}^{2}-4 \Delta_{1}}}{\Delta_{1}}, \Delta_{2}\right. \\
& <-2 \sqrt{\Delta_{1}}, a>0, b>0, c>0, D>0, k>0, D \\
& <k(1-\exp (-b c)),\left(b c-\log \left(\frac{k}{k-D}\right)\right) \\
& \cdot\left(D-(k-D) \log \left(\frac{k}{k-D}\right)\right)<D \\
& \left.\cdot \log \left(\frac{k}{k-D}\right)\right\},
\end{aligned}
$$

$$
\begin{aligned}
F_{3} & =\left\{(a, b, c, D, k, \delta) \mid \delta=\frac{-\Delta_{2}+\sqrt{\Delta_{2}^{2}-4 \Delta_{1}}}{\Delta_{1}}, \Delta_{2}\right. \\
& <-2 \sqrt{\Delta_{1}}, a>0, b>0, c>0, D>0, k>0, D \\
& <k(1-\exp (-b c)),\left(b c-\log \left(\frac{k}{k-D}\right)\right) \\
\cdot & \left(D-(k-D) \log \left(\frac{k}{k-D}\right)\right)<D \\
\cdot & \left.\left.\log \left(\frac{k}{k-D}\right)\right\}, \quad \log \left(\frac{k}{k-D}\right)\right) \\
F_{H} & =\left\{(a, b, c, D, k, \delta) \mid \delta=-\frac{\Delta_{2}}{\Delta_{1}}, \Delta_{2}>-2 \sqrt{\Delta_{1}}, a\right. \\
& >0, b>0, c>0, D>0, k>0, D \\
& <k(1-\exp (-b c)),\left(b c-\log \left(\frac{k}{k-D}\right)\right\} . \\
& \left(D-(k-D) \log \left(\frac{k-D}{k}\right)\right\}
\end{aligned}
$$

From Theorem 4 (4), we see that if $(a, b, c, D, k, \delta) \epsilon$ $F_{2}$ (or $F_{3}$ ) then one of the eigenvalues of the Jacobian matrix $J\left(E_{2}\right)$ at the fixed point $E_{2}\left(x^{*}, y^{*}\right)$ is -1 and the other is neither -1 nor 1 , and if $(a, b, c, D, k, \delta) \in F_{H}$, then the eigenvalues of the matrix $J\left(E_{2}\right)$ are a pair of conjugate complex numbers with modulus 1 .

In the following section, we will investigate a flip bifurcation and Hopf bifurcation of the fixed point $E_{2}\left(x^{*}, y^{*}\right)$ when parameters vary in the small neighborhood of $F_{2}$ (or $\left.F_{3}\right)$ and $F_{H}$, respectively.

\section{Bifurcation Analysis}

In this section, we will discuss two kinds of bifurcation phenomena of system (3), flip bifurcation and Hopf bifurcation, according to the parameter $\delta$. System (3) has no saddle-node bifurcation since the system has at most one positive fixed point $E_{2}\left(x^{*}, y^{*}\right)$ as mentioned in Section 2 .

First we investigate a flip (or period-doubling) bifurcation of system (3) at the fixed point $E_{2}\left(x^{*}, y^{*}\right)$ when parameters vary in a small neighborhood of $F_{2}$. Similar arguments can be applied to the case $F_{3}$.

Taking parameters $\left(a, b, c, D, k, \delta_{1}\right) \in F_{2}$, where $\delta_{1}=$ $\left(-\Delta_{2}-\sqrt{\Delta_{2}^{2}-4 \Delta_{1}}\right) / \Delta_{1}, \Delta_{1}=(a(k-D) / b c)(b c-\log (k /(k-$ $D))) \log (k /(k-D))$, and $\Delta_{2}=(a / b c D)((k-D)(\log (k /(k-$ $\left.D)))^{2}+((b c-2) D-b c k) \log (k /(k-D))+b c D\right)$, we consider system (3) with $\left(a, b, c, D, k, \delta_{1}\right)$ which is described by

$$
\begin{aligned}
& x \longrightarrow x+\delta_{1}\left(a x\left(1-\frac{x}{b}\right)-h(1-\exp (-c x)) y\right), \\
& y \longrightarrow y+\delta_{1}(-D y+k(1-\exp (-c x)) y) .
\end{aligned}
$$


Then system (16) has a unique fixed point $E_{2}\left(x^{*}, y^{*}\right)$ whose eigenvalues are $\lambda_{1}=-1$ and $\lambda_{2}$ with $\left|\lambda_{2}\right| \neq 1$.

Considering a perturbation $\delta^{*}$ at $\delta_{1}$, a perturbed system of system (16) is obtained as follows:

$$
\begin{aligned}
x & \longrightarrow x \\
& +\left(\delta_{1}+\delta^{*}\right)\left(a x\left(1-\frac{x}{b}\right)-h(1-\exp (-c x)) y\right) \\
& \equiv f\left(x, y, \delta^{*}\right)
\end{aligned}
$$

$$
\begin{aligned}
y & \longrightarrow y+\left(\delta_{1}+\delta^{*}\right)(-D y+k(1-\exp (-c x)) y) \\
& \equiv g\left(x, y, \delta^{*}\right),
\end{aligned}
$$

where $\left|\delta^{*}\right| \ll 1$, which is a small perturbation parameter.

In order to transform the fixed point $E_{2}\left(x^{*}, y^{*}\right)$ of system (17) into the origin, let $u=x-x^{*}$ and $v=y-y^{*}$, and then, expanding $f$ and $g$ as a Taylor series at $\left(u, v, \delta^{*}\right)=(0,0,0)$ to the second order, system (17) becomes

$$
\left(\begin{array}{l}
u \\
v
\end{array}\right) \longrightarrow\left(\begin{array}{l}
a_{11} u+a_{12} v+a_{13} u v+a_{14} u^{2}+e_{1} \delta^{*}+e_{2} u \delta^{*}+e_{3} v \delta^{*}+O\left(\left(|u|+|v|+\left|\delta^{*}\right|\right)^{3}\right) \\
a_{21} u+a_{22} v+a_{23} u v+a_{24} u^{2}+e_{4} \delta^{*}+e_{5} u \delta^{*}+e_{6} v \delta^{*}+O\left(\left(|u|+|v|+\left|\delta^{*}\right|\right)^{3}\right)
\end{array}\right)
$$

where

$$
\begin{aligned}
& a_{11}=1+a \delta_{1}-\frac{2 a \delta_{1} x^{*}}{b}-c \delta_{1} h y^{*} \exp \left(-c x^{*}\right) \\
& a_{12}=\delta_{1} h \exp \left(-c x^{*}\right) \\
& a_{13}=-c \delta_{1} h \exp \left(-c x^{*}\right) \text {, } \\
& a_{14}=-\frac{a \delta_{1}}{b}+\frac{1}{2} c^{2} \delta_{1} h y^{*} \exp \left(-c x^{*}\right), \\
& a_{21}=c \delta_{1} k y^{*} \exp \left(-c x^{*}\right) \text {, } \\
& a_{22}=1-D \delta_{1}-\delta_{1} k \exp \left(-c x^{*}\right), \\
& a_{23}=c k \delta_{1} \exp \left(-c x^{*}\right) \text {, } \\
& a_{24}=-\frac{1}{2} c^{2} \delta_{1} k y^{*} \exp \left(-c x^{*}\right) \text {, } \\
& e_{1}=-h+a x^{*}-\frac{a x^{* 2}}{b}+h y^{*} \exp \left(-c x^{*}\right) \text {, } \\
& e_{2}=a-\frac{2 a x^{*}}{b}-c h y^{*} \exp \left(-c x^{*}\right), \\
& e_{3}=h \exp \left(-c x^{*}\right) \text {, } \\
& e_{4}=k-D y^{*}-k y^{*} \exp \left(-c x^{*}\right), \\
& e_{5}=c k y^{*} \exp \left(-c x^{*}\right) \text {, } \\
& e_{6}=-D-k \exp \left(-c x^{*}\right) \text {. }
\end{aligned}
$$

We construct an invertible matrix

$$
T=\left(\begin{array}{cc}
a_{12} & a_{12} \\
-1-a_{11} & \lambda_{2}-a_{11}
\end{array}\right)
$$

and using the transformation

$$
\left(\begin{array}{l}
u \\
v
\end{array}\right)=T\left(\begin{array}{l}
\bar{x} \\
\bar{y}
\end{array}\right)
$$

for system (18), then system (18) can be written as

$$
\left(\begin{array}{l}
\bar{x} \\
\bar{y}
\end{array}\right)=\left(\begin{array}{cc}
-1 & 0 \\
0 & \lambda_{2}
\end{array}\right)\left(\begin{array}{l}
\bar{x} \\
\bar{y}
\end{array}\right)+\left(\begin{array}{l}
f_{1}\left(\bar{x}, \bar{y}, \delta^{*}\right) \\
g_{1}\left(\bar{x}, \bar{y}, \delta^{*}\right)
\end{array}\right)
$$

where

$$
\begin{aligned}
f_{1}\left(\bar{x}, \bar{y}, \delta^{*}\right)= & \frac{\left(\lambda_{2}-a_{11}\right) a_{14}-a_{12} a_{24}}{a_{12}\left(1+\lambda_{2}\right)} u^{2} \\
& +\frac{\left(\lambda_{2}-a_{11}\right) a_{13}-a_{12} a_{23}}{a_{12}\left(1+\lambda_{2}\right)} u v \\
& +\frac{\left(\lambda_{2}-a_{11}\right) e_{2}-a_{12} e_{5}}{a_{12}\left(1+\lambda_{2}\right)} u \delta^{*} \\
& +\frac{\left(\lambda_{2}-a_{11}\right) e_{3}-a_{12} e_{6}}{a_{12}\left(1+\lambda_{2}\right)} v \delta^{*} \\
& +\frac{\left(\lambda_{2}-a_{11}\right) e_{1}-a_{12} e_{4}}{a_{12}\left(1+\lambda_{2}\right)} \delta^{*} \\
& +O\left(|u|+|v|+\left|\delta^{*}\right|\right)^{3}, \\
g_{1}\left(\bar{x}, \bar{y}, \delta^{*}\right)= & \frac{\left(a_{11}+1\right) a_{14}+a_{12} a_{24}}{a_{12}\left(1+\lambda_{2}\right)} u^{2} \\
& +\frac{\left(a_{11}+1\right) a_{13}+a_{12} a_{23}}{a_{12}\left(1+\lambda_{2}\right)} u v \\
& +\frac{\left(a_{11}+1\right) e_{2}+a_{12} e_{5}}{a_{12}\left(1+\lambda_{2}\right)} u \delta^{*} \\
& O(1+1)
\end{aligned}
$$




$$
\begin{aligned}
& +\frac{\left(a_{11}+1\right) e_{3}+a_{12} e_{6}}{a_{12}\left(1+\lambda_{2}\right)} v \delta^{*} \\
& +\frac{\left(a_{11}+1\right) e_{1}+a_{12} e_{4}}{a_{12}\left(1+\lambda_{2}\right)} \delta^{*} \\
& +O\left(|u|+|v|+\left|\delta^{*}\right|\right)^{3} .
\end{aligned}
$$

And $u=a_{12}(\bar{x}+\bar{y}), v=-\left(1+a_{11}\right) \bar{x}+\left(\lambda_{2}-a_{11}\right) \bar{y}$.
It follows from the center manifold theorem [32] that there exists a center manifold $W^{c}(0,0)$ of system $(18)$ at the fixed point $(0,0)$ in a small neighborhood of $\delta^{*}=0$, which can be approximately represented as follows:

$$
\begin{aligned}
& W^{\mathcal{c}}(0,0)=\left\{(\bar{x}, \bar{y}) \mid \bar{y}=r_{1} \bar{x}^{2}+r_{2} \bar{x} \delta^{*}+r_{3} \delta^{* 2}\right. \\
& \left.\quad+O\left(\left(|\bar{x}|+\left|\delta^{*}\right|\right)^{3}\right)\right\}
\end{aligned}
$$

where

$$
\begin{aligned}
r_{1}= & \frac{\left(1+a_{11}\right)^{2} a_{13}-a_{12}\left(\left(1+a_{11}\right) a_{14}-\left(1+a_{11}\right) a_{23}+a_{12} a_{24}\right)}{\left(\lambda_{2}^{2}-1\right)}, \\
r_{2}= & \frac{\left(1+a_{11}\right)^{2} e_{3}-a_{12}^{2} e_{5}-\left(1+a_{11}\right) a_{12}\left(e_{2}-e_{6}\right)}{a_{12}\left(\lambda_{2}+1\right)^{2}} \\
& +\frac{2\left(\left(1+a_{11}\right)^{2} a_{13}-a_{12}\left(\left(1+a_{11}\right) a_{14}-\left(1+a_{11}\right) a_{23}+a_{12} a_{24}\right)\right)\left(a_{11} e_{1}+a_{12} e_{4}-e_{1} \lambda_{2}\right)}{a_{12}\left(\lambda_{2}-1\right)\left(\lambda_{2}+1\right)^{3}}, \\
r_{3}= & \frac{\left(-\left(1+a_{11}\right)^{2} e_{3}+a_{12}^{2} e_{5}+\left(1+a_{11}\right) a_{12}\left(e_{2}-e_{6}\right)\right)\left(a_{11} e_{1}+a_{12} e_{4}-e_{1} \lambda_{2}\right)}{a_{12}^{2}\left(\lambda_{2}-1\right)\left(\lambda_{2}+1\right)^{3}} \\
& +\frac{\left(\left(1+a_{11}\right)^{2} a_{13}-a_{12}\left(\left(1+a_{11}\right) a_{14}-\left(1+a_{11}\right) a_{23}+a_{12} a_{24}\right)\right)\left(a_{11} e_{1}+a_{12} e_{4}-e_{1} \lambda_{2}\right)^{2}}{a_{12}^{2}\left(\lambda_{2}-1\right)\left(\lambda_{2}+1\right)^{4}} .
\end{aligned}
$$

Thus we can obtain the map, which is restricted to the center manifold $W^{c}(0,0)$ :

$$
\begin{aligned}
G: \bar{x} \longrightarrow & p_{1} \delta^{*}+p_{2} \bar{x}^{2}+p_{3} \bar{x} \delta^{*}+p_{4} x \delta^{* 2}+p_{5} x^{2} \delta^{*} \\
& +p_{6} \bar{x}^{3}+p_{7} \delta^{* 3}+O\left(\left(|\bar{x}|+\left|\delta^{*}\right|\right)^{4}\right),
\end{aligned}
$$

where

$$
\begin{aligned}
p_{1}= & h_{1}, \\
p_{2}= & a_{12}^{2} h_{1}-\left(a_{11}+1\right) a_{12} h_{2}, \\
p_{3}= & a_{12} h_{3}-\left(a_{11}+1\right) h_{4}, \\
p_{4}= & 2 a_{12}^{2} r_{3} h_{1}-\left(a_{11}+1\right) a_{12} r_{3} h_{2}+a_{12} r_{2} h_{3} \\
& +a_{12} r_{3} h_{2}\left(\lambda_{2}-a_{11}\right)+r_{2} h_{4}\left(\lambda_{2}-a_{11}\right), \\
p_{5}= & 2 a_{12}^{2} r_{2} h_{1}-\left(a_{11}+1\right) a_{12} r_{2} h_{2}+r_{1} a_{12} h_{3} \\
& +a_{12} r_{2} h_{2}\left(\lambda_{2}-a_{11}\right)+r_{1} h_{4}\left(\lambda_{2}-a_{11}\right), \\
p_{6}= & 2 r_{1} a_{12}^{2} h_{1}-r_{1}\left(a_{11}+1\right) a_{12} h_{2} \\
& +r_{1} a_{12} h_{2}\left(\lambda_{2}-a_{11}\right), \\
p_{7}= & r_{3} a_{12} h_{3}, \\
h_{1}= & \frac{\left(\lambda_{2}-a_{11}\right) a_{14}-a_{12} a_{24}}{a_{12}\left(1+\lambda_{2}\right)},
\end{aligned}
$$

$$
\begin{aligned}
& h_{2}=\frac{\left(\lambda_{2}-a_{11}\right) a_{13}-a_{12} a_{23}}{a_{12}\left(1+\lambda_{2}\right)}, \\
& h_{3}=\frac{\left(\lambda_{2}-a_{11}\right) e_{2}-a_{12} e_{5}}{a_{12}\left(1+\lambda_{2}\right)}, \\
& h_{4}=\frac{\left(\lambda_{2}-a_{11}\right) e_{3}-a_{12} e_{6}}{a_{12}\left(1+\lambda_{2}\right)}, \\
& h_{5}=\frac{\left(\lambda_{2}-a_{11}\right) e_{1}-a_{12} e_{4}}{a_{12}\left(1+\lambda_{2}\right)} .
\end{aligned}
$$

In order for the map (26) to undergo flip bifurcation, we require that two discriminatory quantities $\alpha_{1}$ and $\alpha_{2}$ are not zero, where

$$
\begin{aligned}
& \alpha_{1}=\left.\left(\frac{\partial^{2} G}{\partial \bar{x} \partial \delta^{*}}+\frac{1}{2} \frac{\partial G \partial^{2} G}{\partial \delta^{*} \partial \bar{x}^{2}}\right)\right|_{(0,0)}=p_{1} p_{2} \\
& \alpha_{2}=\left.\left(\frac{1}{6} \frac{\partial^{3} G}{\partial \bar{x}^{3}}+\left(\frac{1}{2} \frac{\partial^{2} G}{\partial \bar{x}^{2}}\right)^{2}\right)\right|_{(0,0)}=p_{2}^{2}+p_{6}
\end{aligned}
$$

From the above analysis and the theorems in [32] we have the following theorem.

Theorem 5. If $\alpha_{1} \neq 0$ and $\alpha_{2} \neq 0$, then system (3) undergoes a flip bifurcation at the fixed point $E_{2}\left(x^{*}, y^{*}\right)$ when $\delta$ varies 
in a small neighborhood of $\delta^{*}$. Moreover, if $\alpha_{2}>0$ (resp. $\alpha_{2}<$ $0)$, then the period-2 points that bifurcate from $E_{2}\left(x^{*}, y^{*}\right)$ are stable (resp. unstable).

Next we discuss Hopf bifurcation of system (3) at $E_{2}\left(x^{*}, y^{*}\right)$ when parameters of system (3) vary in a small neighborhood of $F_{H}$. For this, taking parameters $\left(a, b, c, D, k, \delta_{2}\right) \in F_{H}$, where $\delta_{2}=-\Delta_{2} / \Delta_{1}, \Delta_{1}=(a(k-$ $D) / b c)(b c-\log (k /(k-D))) \log (k /(k-D))$, and $\Delta_{2}=$ $(a / b c D)\left((k-D)(\log (k /(k-D)))^{2}+((b c-2) D-b c k) \log (k /(k-\right.$ $D))+b c D)$, we consider system (3) with $\left(a, b, c, D, k, \delta_{2}\right)$ which is described by

$$
\begin{aligned}
& x \longrightarrow x+\delta_{2}\left(a x\left(1-\frac{x}{b}\right)-h(1-\exp (-c x)) y\right), \\
& y \longrightarrow y+\delta_{2}(-D y+k(1-\exp (-c x)) y) .
\end{aligned}
$$

Then it follows from Theorem 4 that the roots of the characteristic equation (8) associated with system (30) at $E_{2}\left(x^{*}, y^{*}\right)$ are a pair of conjugate complex numbers with modulus 1 .

Now consider a perturbation system of system (30) by choosing the bifurcation parameter $\delta^{* *}$ as follows:

$$
\begin{aligned}
x & \longrightarrow x \\
& +\left(\delta_{2}+\delta^{* *}\right)\left(a x\left(1-\frac{x}{b}\right)-h(1-\exp (-c x)) y\right), \\
y & \longrightarrow y+\left(\delta_{2}+\delta^{* *}\right)(-D y+k(1-\exp (-c x)) y),
\end{aligned}
$$

where $\left|\delta^{* *}\right| \ll 1$, which is a small perturbation parameter.

Let $u_{1}=x-x^{*}$ and $v_{1}=y-y^{*}$ in system (31) to transform the equilibrium $E_{2}\left(x^{*}, y^{*}\right)$ into the origin. Thus system (31) can be rewritten as

$$
\left(\begin{array}{l}
u_{1} \\
v_{1}
\end{array}\right) \longrightarrow\left(\begin{array}{l}
f_{2}\left(u_{1}, v_{1}, \delta^{* *}\right) \\
g_{2}\left(u_{1}, v_{1}, \delta^{* *}\right)
\end{array}\right)
$$

where

$$
\begin{aligned}
& f_{2}\left(u_{1}, v_{1}, \delta^{* *}\right)=u_{1} \\
& \quad+\frac{\delta_{2}+\delta^{* *}}{b}\left(a\left(b-u_{1}-x^{*}\right)\left(u_{1}+x^{*}\right)\right. \\
& \left.\quad-b h\left(v_{1}+y^{*}\right)+b h\left(v_{1}+y^{*}\right) \exp \left(-c\left(u_{1}+x^{*}\right)\right)\right), \\
& g_{2}\left(u_{1}, v_{1}, \delta^{* *}\right)=v_{1}+\left(\delta_{2}+\delta^{* *}\right)\left(v_{1}+y^{*}\right)(-D \\
& \left.\quad+k\left(1-\exp \left(-c\left(u_{1}+x^{*}\right)\right)\right)\right) .
\end{aligned}
$$

Note that the characteristic equation associated with the linearization of the map $(32)$ at $\left(u_{1}, v_{1}\right)=(0,0)$ is given by

$$
\lambda^{2}-p\left(\delta^{* *}\right) \lambda+q\left(\delta^{* *}\right)=0
$$

where

$$
\begin{aligned}
& p\left(\delta^{* *}\right)=2+\Delta_{2}\left(\delta_{2}+\delta^{* *}\right), \\
& q\left(\delta^{* *}\right)=1+\Delta_{1}\left(\delta_{2}+\delta^{* *}\right)^{2}+\Delta_{2}\left(\delta_{2}+\delta^{* *}\right), \\
& \Delta_{1}=\frac{a(k-D)}{b c}\left(b c-\log \left(\frac{k}{k-D}\right)\right) \log \left(\frac{k}{k-D}\right), \\
& \Delta_{2}=\frac{a}{b c D}\left((k-D)\left(\log \left(\frac{k}{k-D}\right)\right)^{2}\right. \\
& \left.\quad+((b c-2) D-b c k) \log \left(\frac{k}{k-D}\right)+b c D\right) .
\end{aligned}
$$

The roots of the characteristic equation (35) are

$$
\lambda, \bar{\lambda}=\frac{1}{2}\left(p\left(\delta^{* *}\right) \pm \sqrt{p\left(\delta^{* *}\right)^{2}-4 q\left(\delta^{* *}\right)}\right)
$$

and then we have

$$
|\lambda|=\left(q\left(\delta^{* *}\right)\right)^{1 / 2}
$$

Also, it follows from Theorem 4 that $|\lambda|=|\bar{\lambda}|=1$ when $\delta^{* *}=0$. Since $\left(a, b, c, D, k, \delta_{2}\right) \in F_{H}$, we have $\Delta_{1} \delta_{2}=-\Delta_{2}$. Therefore,

$$
l=\left.\frac{d|\lambda|}{d \delta^{* *}}\right|_{\delta^{* *}=0}=-\frac{1}{2} \Delta_{2}>0 .
$$

In addition, it is required that, when $\delta^{* *}=0, \lambda^{m}, \bar{\lambda}^{m} \neq 1$ for $m=1,2,3,4$, which is equivalent to $p(0) \neq-2,0,1,2$. Since $p^{2}(0)-4 q(0)<0$ and $q(0)=1, p(0) \neq-2,2$. Thus we only show that $p(0) \neq 0,1$, which leads to $p(0)=2+\Delta_{2} \delta_{2} \neq$ 0,1 . Then $-\Delta_{2} \delta_{2} \neq 1,2$ and therefore

$$
\begin{aligned}
& -\frac{a \delta_{2}}{b c D}\left((k-D)\left(\log \left(\frac{k}{k-D}\right)\right)^{2}\right. \\
& \left.-\delta_{2}((b c-2) D-b c k) \log \left(\frac{k}{k-D}\right)+b c D\right) \\
& \quad \neq 1,2 .
\end{aligned}
$$

Hence the eigenvalues $\lambda, \bar{\lambda}$ do not lie in the intersection of the unit circle with the coordinate axes when $\delta^{* *}=0$ and (40) holds.

Next we investigate the normal form of system (32) when $\delta^{* *}=0$. Let

$$
\begin{aligned}
\alpha= & 1+\frac{1}{2} \delta_{2}\left(\frac { a } { b c D } \left((k-D)\left(\log \left(\frac{k}{k-D}\right)\right)^{2}\right.\right. \\
& \left.\left.+((b c-2) D-b c k) \log \left(\frac{k}{k-D}\right)+b c D\right)\right),
\end{aligned}
$$

$\beta=\frac{1}{2} \delta_{2} \sqrt{4 \Delta_{1}-\Delta_{2}^{2}}$

$$
T=\left(\begin{array}{ll}
0 & 1 \\
\beta & \alpha
\end{array}\right) \text {. }
$$


By expanding $f_{2}$ and $g_{2}$ in system (32) as Taylor series at $\left(u_{1}, v_{1}\right)=(0,0)$ to the third order and use the transformation

$$
\left(\begin{array}{l}
u_{1} \\
v_{1}
\end{array}\right)=T\left(\begin{array}{l}
\tilde{x} \\
\tilde{y}
\end{array}\right)
$$

for system (32), system (32) becomes

$$
\left(\begin{array}{l}
\tilde{x} \\
\tilde{y}
\end{array}\right)=\left(\begin{array}{cc}
\alpha & -\beta \\
\beta & \alpha
\end{array}\right)\left(\begin{array}{l}
\tilde{x} \\
\tilde{y}
\end{array}\right)+\left(\begin{array}{l}
P(\tilde{x}, \tilde{y}) \\
Q(\tilde{x}, \tilde{y})
\end{array}\right),
$$

where

$$
\begin{aligned}
& P(\tilde{x}, \tilde{y})=\frac{\alpha}{\beta}\left(\tilde{a}_{23}-\alpha \tilde{a}_{13}\right) \tilde{x} \tilde{y} \\
& +\left(-\alpha \widetilde{a}_{13}+\widetilde{a}_{23}+\frac{1}{\beta}\left(\widetilde{a}_{24}-\alpha \widetilde{a}_{14}\right)\right) \tilde{x}^{2} \\
& +\frac{\alpha}{\beta}\left(\tilde{a}_{25}-\alpha \tilde{a}_{15}\right) \tilde{x}^{2} \tilde{y} \\
& +\left(-\alpha \widetilde{a}_{15}+\tilde{a}_{25}+\frac{1}{\beta}\left(\tilde{a}_{26}-\alpha \widetilde{a}_{16}\right)\right) \tilde{x}^{3} \\
& +O\left((|\widetilde{x}|+|\tilde{y}|)^{4}\right), \\
& Q(\tilde{x}, \tilde{y})=\alpha \widetilde{a}_{13} \tilde{x} \tilde{y}+\left(\widetilde{a}_{14}+\beta \widetilde{a}_{13}\right) \tilde{x}^{2}+\alpha \widetilde{a}_{15} \tilde{x}^{2} \tilde{y} \\
& +\left(\tilde{a}_{16}+\beta \widetilde{a}_{15}\right) \tilde{x}^{3}+O\left((|\tilde{x}|+|\tilde{y}|)^{4}\right), \\
& \tilde{a}_{13}=-c \delta_{2} h \exp \left(-c x^{*}\right) \text {, } \\
& \tilde{a}_{14}=-\frac{a \delta_{2}}{b}+\frac{1}{2} c^{2} \delta_{1} h y^{*} \exp \left(-c x^{*}\right), \\
& \tilde{a}_{15}=\frac{1}{2} c^{2} \delta_{2} h y^{*} \exp \left(-c x^{*}\right) \text {, } \\
& \tilde{a}_{16}=-\frac{1}{6} c^{3} \delta_{2} h y^{*} \exp \left(-c x^{*}\right) \text {, } \\
& \tilde{a}_{23}=c k \delta_{2} * \exp \left(-c x^{*}\right) \text {, } \\
& \tilde{a}_{24}=-\frac{1}{2} c^{2} \delta_{2} k y^{*} \exp \left(-c x^{*}\right) \text {, } \\
& \tilde{a}_{25}=-\frac{1}{2} c^{2} \delta_{2} k \exp \left(-c x^{*}\right) \text {, } \\
& \tilde{a}_{26}=\frac{1}{6} c^{3} \delta_{2} k y^{*} \exp \left(-c x^{*}\right) \text {. }
\end{aligned}
$$

In order to undergo Hopf bifurcation, it is required that the following discriminatory quantity $A$ is not zero [33]:

$$
\begin{aligned}
A= & -\operatorname{Re}\left[\frac{(1-2 \lambda) \bar{\lambda}^{2}}{1-\lambda} \xi_{11} \xi_{20}\right]-\frac{1}{2}\left\|\xi_{11}\right\|^{2}-\left\|\xi_{02}\right\|^{2} \\
& +\operatorname{Re}\left(\bar{\lambda} \xi_{21}\right)
\end{aligned}
$$

where

$$
\begin{aligned}
& \xi_{20}=\frac{1}{8}\left[P_{\tilde{x} \tilde{x}}(0,0)-P_{\tilde{y} \tilde{y}}(0,0)+2 Q_{\tilde{x} \tilde{y}}(0,0)\right. \\
& \left.+i\left(Q_{\tilde{x} \tilde{x}}(0,0)-Q_{\tilde{y} \tilde{y}}(0,0)-2 P_{\tilde{x} \tilde{y}}(0,0)\right)\right] \\
& =\frac{1}{4}\left[\alpha \widetilde{a}_{13}-\frac{\alpha}{\beta}\left(\beta \widetilde{a}_{13}+\tilde{a}_{14}\right)+\frac{\tilde{a}_{24}}{\beta}+\tilde{a}_{23}+i\left(\beta \widetilde{a}_{13}\right.\right. \\
& \left.\left.+\tilde{a}_{14}+\frac{\alpha}{\beta}\left(\alpha \tilde{a}_{13}-\tilde{a}_{23}\right)\right)\right], \\
& \xi_{11}=\frac{1}{4}\left[P_{\tilde{x} \tilde{x}}(0,0)+P_{\tilde{y} \tilde{y}}(0,0)+i\left(Q_{\tilde{x} \tilde{x}}(0,0)\right.\right. \\
& \left.\left.+Q_{\tilde{x} \tilde{y}}(0,0)\right)\right]=\frac{1}{2}\left[-\frac{\alpha}{\beta}\left(\beta \widetilde{a}_{13}+\tilde{a}_{14}+\frac{\tilde{a}_{24}}{\beta}+\tilde{a}_{23}\right)\right. \\
& \left.+i \frac{1}{4}\left((\alpha+2 \beta) \tilde{a}_{13}+2 \tilde{a}_{14}\right)\right] \text {, } \\
& \xi_{02}=\frac{1}{8}\left[P_{\tilde{x} \tilde{x}}(0,0)-P_{\tilde{y} \tilde{y}}(0,0)-2 Q_{\tilde{x} \tilde{y}}(0,0)\right. \\
& \left.+i\left(Q_{\tilde{x} \tilde{x}}(0,0)-Q_{\tilde{y} \tilde{y}}(0,0)+2 P_{\tilde{x} \tilde{y}}(0,0)\right)\right] \\
& =\frac{1}{4}\left[-\alpha \widetilde{a}_{13}-\frac{\alpha}{\beta}\left(\beta \widetilde{a}_{13}+\widetilde{a}_{14}\right)+\frac{\widetilde{a}_{24}}{\beta}+\widetilde{a}_{23}\right. \\
& \left.+i\left(\beta \tilde{a}_{13}+\tilde{a}_{14}-\frac{\alpha}{\beta}\left(\alpha \tilde{a}_{13}-\tilde{a}_{23}\right)\right)\right], \\
& \xi_{21}=\frac{1}{16}\left[P_{\tilde{x} \tilde{x} \tilde{x}}(0,0)+P_{\tilde{x} \tilde{y} \tilde{y}}(0,0)+Q_{\tilde{x} \tilde{x} \tilde{y}}(0,0)\right. \\
& +Q_{\tilde{y} \tilde{y} \tilde{y}}(0,0)+i\left(Q_{\tilde{x} \tilde{x} \tilde{x}}(0,0)+Q_{\tilde{x} \tilde{y} \tilde{y}}(0,0)\right. \\
& \left.\left.-P_{\tilde{x} \tilde{x} \tilde{y}}(0,0)-P_{\tilde{y} \tilde{y} \tilde{y}}(0,0)\right)\right]=\frac{1}{8}\left[\alpha \widetilde{a}_{15}-\frac{3 \alpha}{\beta}\left(\beta \widetilde{a}_{15}\right.\right. \\
& \left.+\tilde{a}_{16}\right)+3\left(\tilde{a}_{25}+\frac{\widetilde{a}_{26}}{\beta}\right)+i\left(3\left(\beta \tilde{a}_{15}+\tilde{a}_{16}\right)\right. \\
& \left.\left.+\frac{\alpha}{\beta}\left(\alpha \tilde{a}_{15}-\tilde{a}_{25}\right)\right)\right] .
\end{aligned}
$$

From the above analysis and theorems in [33], we have the following result.

Theorem 6. If condition (40) holds and $A \neq 0$, then system (3) undergoes Hopf bifurcation at the fixed point $E_{2}\left(x^{*}, y^{*}\right)$ when the parameter $\delta^{* *}$ varies in the small neighborhood of the origin. Moreover, if $A<0$ (resp., $A>0$ ), then an attracting (resp., repelling) invariant closed curve bifurcates from $E_{2}\left(x^{*}, y^{*}\right)$ for $\delta^{* *}>0$ (resp., $\left.\delta^{* *}<0\right)$.

\section{Numerical Simulations}

In this section, we present some bifurcation diagrams and phase portraits of system (3) to substantiate the above theoretical results and illustrate various dynamical behaviors 


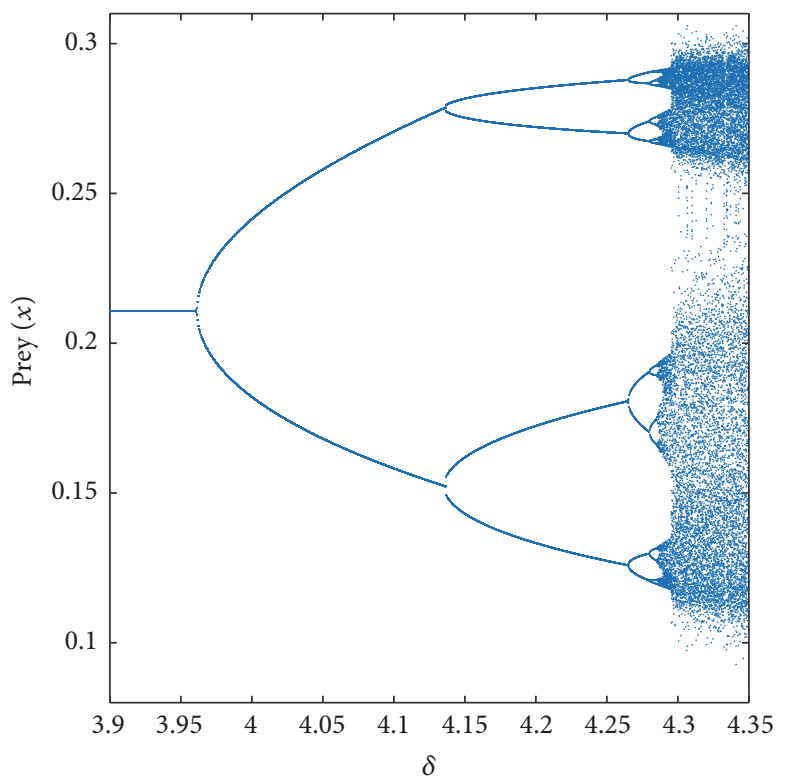

(a)

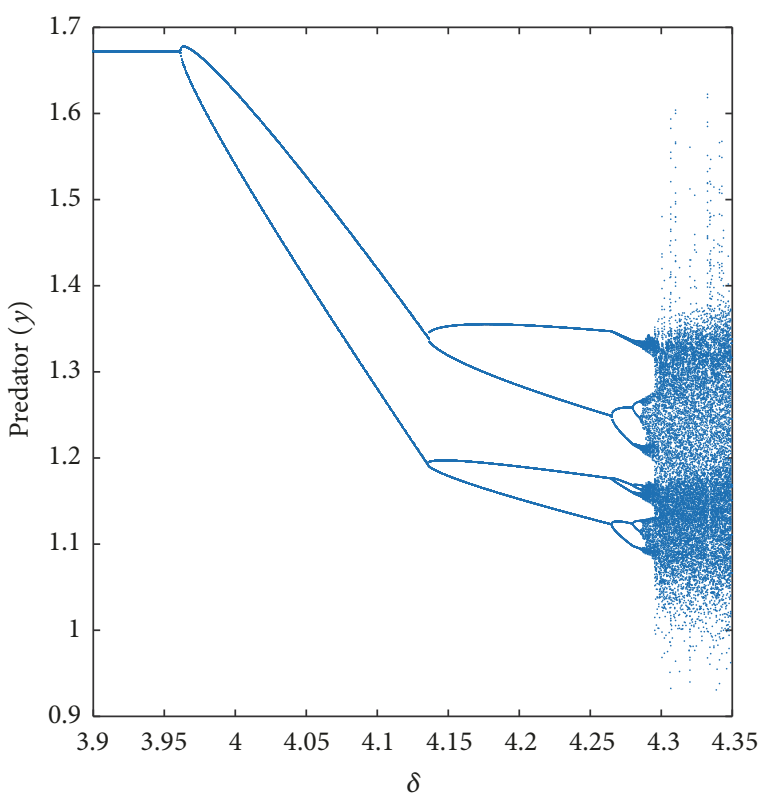

(b)

Figure 1: (a) Bifurcation diagram of system (3) in the $(\delta, x)$ plane when $a=0.8, b=0.3, c=0.5, D=0.1, h=0.3, k=1$, and $3.9 \leq \delta \leq 4.35$. The initial value is $(0.2117,1.6733)$. (b) Bifurcation diagram of system $(3)$ in the $(\delta, y)$ plane.

via numerical simulations. In particular, we calculate the maximum Lyapunov exponents numerically to show the dynamical complexity of system (3). The bifurcation parameters are considered in the following two cases:

(i) Varying $\delta$ in range $3.9 \leq \delta \leq 4.35$, and fixing $a=0.8$, $b=0.3, c=0.5, D=0.1, h=0.3, k=1$.

(ii) Varying $\delta$ in range $2.35 \leq \delta \leq 4.15$, and fixing $a=0.1$, $b=1, c=1, D=0.4, h=0.6, k=1$.

For case (i), based on Theorem 4, we can know that system (4) has only one positive fixed point $E_{2}\left(x^{*}, y^{*}\right)=$ $(0.2107,1.6723)$ since $D<k(1-\exp (-b c))=0.1393$ and $(b c-\log (k /(k-D)))(D-(k-D) \log (k /(k-D)))-D \log (k /(k-$ $D))=-0.0103<0$. It is obtained from tedious calculation that $\alpha_{1}=p_{1} p_{2}=121.9182$ and $\alpha_{2}=p_{2}^{2}+p_{6}=21.2815$ in (28) and (29), respectively. Thus it follows from Theorem 5 that a flip bifurcation emerges from the fixed point $E_{2}$ at $\delta_{1}=$ 3.9613 when $\left(a, b, c, D, k, \delta_{1}\right)=(0.8,0.3,0.5,0.1,1,3.9613)$ in $F_{2}$. The bifurcation diagrams of system (3) are given in Figures 1(a) and 1(b) for showing this phenomenon. From Figure 1, we can observe that the fixed point $E_{2}$ of system (3) is stable for $\delta<3.9613$ and loses its stability at $\delta=3.9613$. We can also see that there is a cascade of period-doubling due to the flip bifurcation. In fact, there are orbits of period $1,2,4,8$ for $\delta \in(3.9,4.27)$, which is illustrated in Figure 3. Moreover, chaotic phase portraits can be observed as shown in Figure 4. In order to confirm the existence of chaotic trajectories of system (3), the maximum Lyapunov exponents corresponding to Figure 1 are computed in Figure 2.

For case (ii), it follows from Theorem 4 that system (4) has only one positive fixed point $E_{2}\left(x^{*}, y^{*}\right)=(0.5108,0.1041)$, which is sink when $\delta<\delta_{2}=-\Delta_{2} / \Delta_{1}=2.6444$, since

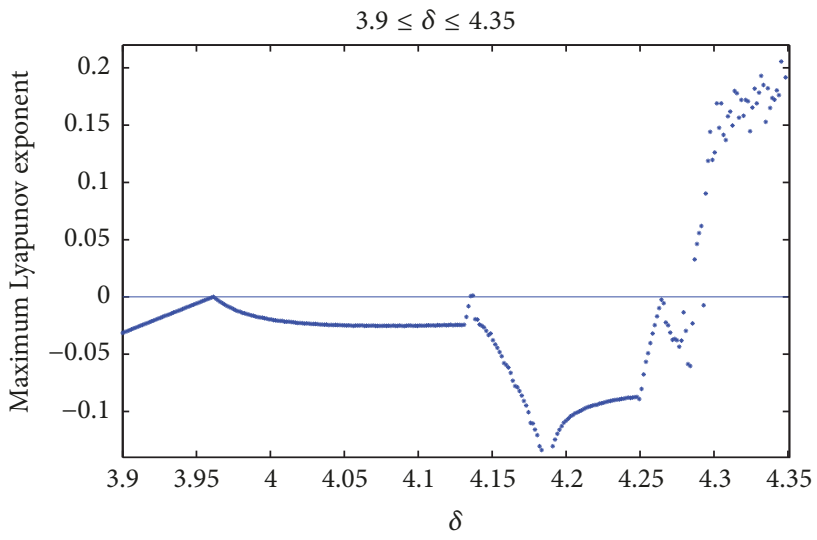

FIGURE 2: Maximum Lyapunov exponents of system (3) corresponding to Figure 1.

$D<k(1-\exp (-b c))=0.6321,(b c-\log (k /(k-D)))(D-$ $(k-D) \log (k /(k-D)))-D \log (k /(k-D))=-0.1586<0$, and $\Delta_{2}<-2 \sqrt{\Delta_{1}}$. After elementary calculation, we can obtain that $-\left(a \delta_{2} / b c D\right)\left((k-D)(\log (k /(k-D)))^{2}-\delta_{2}((b c-\right.$ 2) $D-b c k) \log (k /(k-D))+b c D)=-1.6182$ in $(40)$ and $A=-3.4722$ in (45), which means that the hypotheses of Theorem 6 hold. Thus from Theorem 6, Hopf bifurcation emerges from the fixed point $(0.5108,0.1041)$ at $\delta_{2}=2.6444$. In order to investigate various dynamical phenomena for this case, the bifurcation diagrams of system (3) are displayed in Figures 5(a) and 5(c). It is observed from Figure 5(a) that the fixed point $(0.5108,0.1041)$ of system (3) is sink for $\delta<\delta_{2}$, and an attracting invariant close curve appears, as shown in Figure 7, when the parameter $\delta$ exceeds $\delta_{2}$. After 


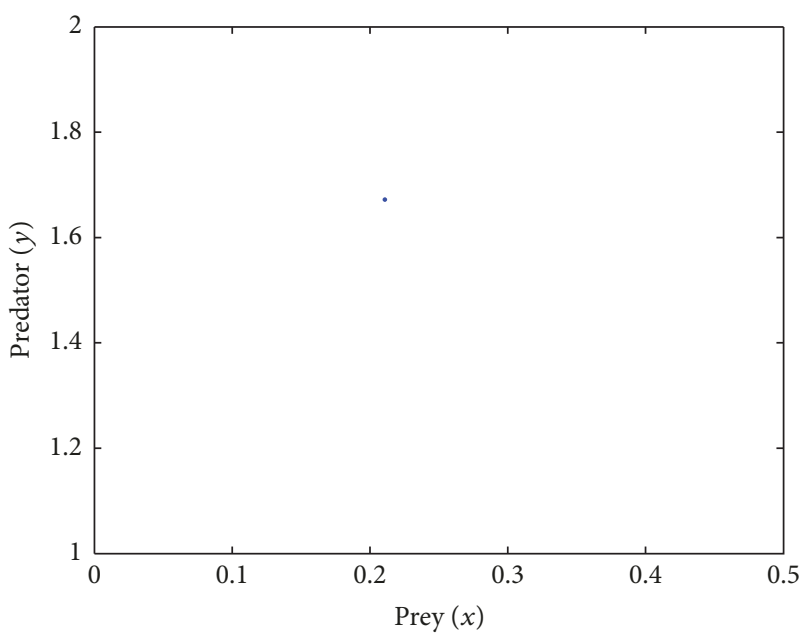

(a) $\delta=3.95$

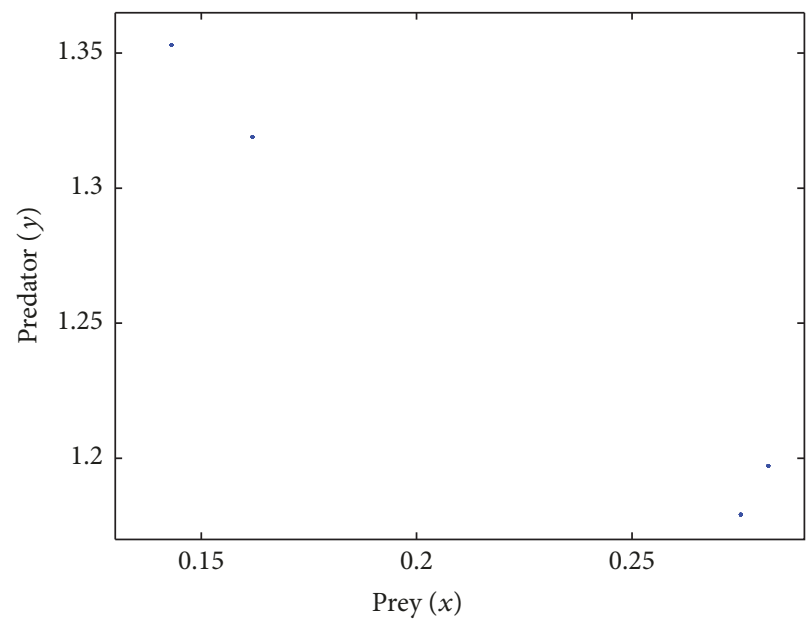

(c) $\delta=4.15$

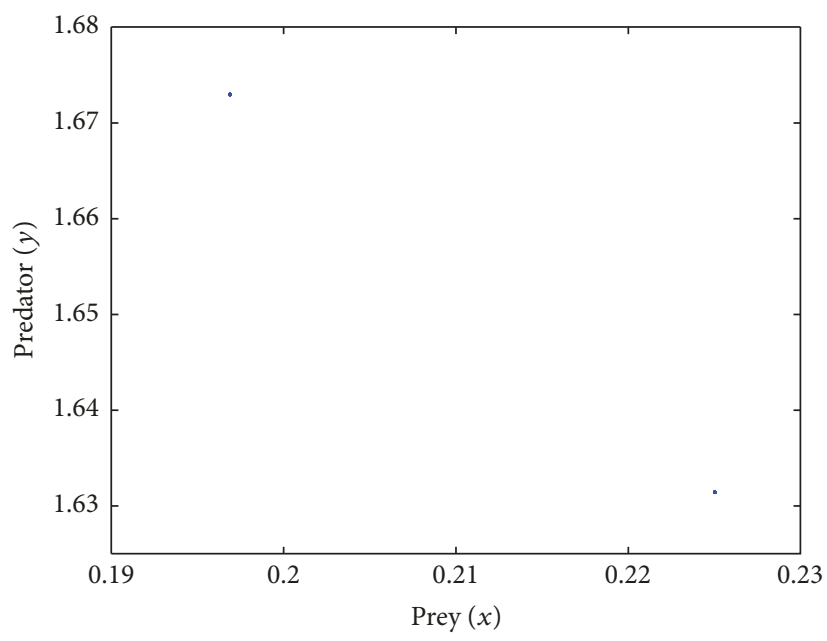

(b) $\delta=3.97$

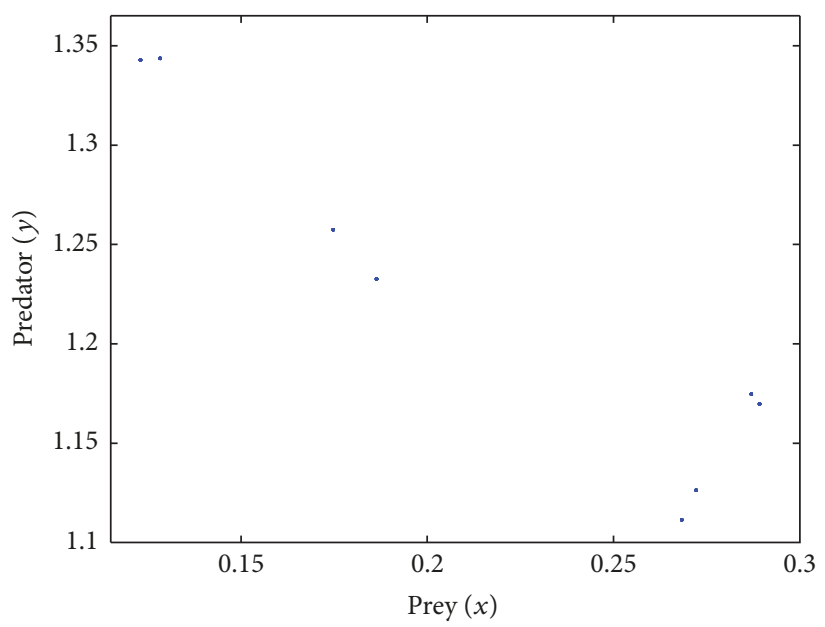

(d) $\delta=4.27$

Figure 3: (a) Phase portrait of a period-1 orbit of system (3) when $a=0.8, b=0.3, c=0.5, D=0.1, h=0.3, k=1$, and $\delta=3.95$. (b) Phase portrait of a period- 2 orbit when $\delta=3.97$. (c) Phase portrait of a period- 4 orbit when $\delta=4.15$. (d) Phase portrait of a period- 8 orbit when $\delta=4.27$.

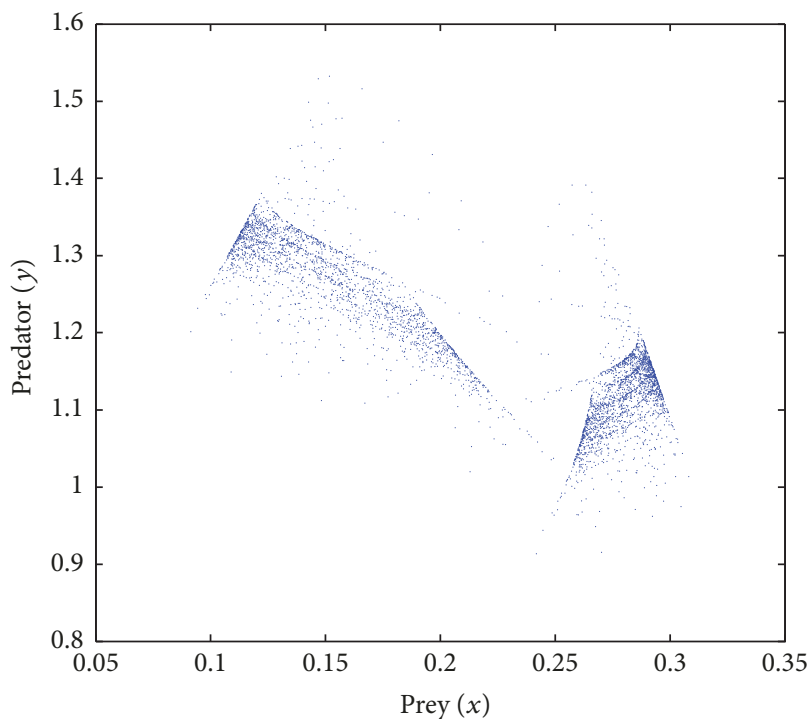

(a) $\delta=4.33$

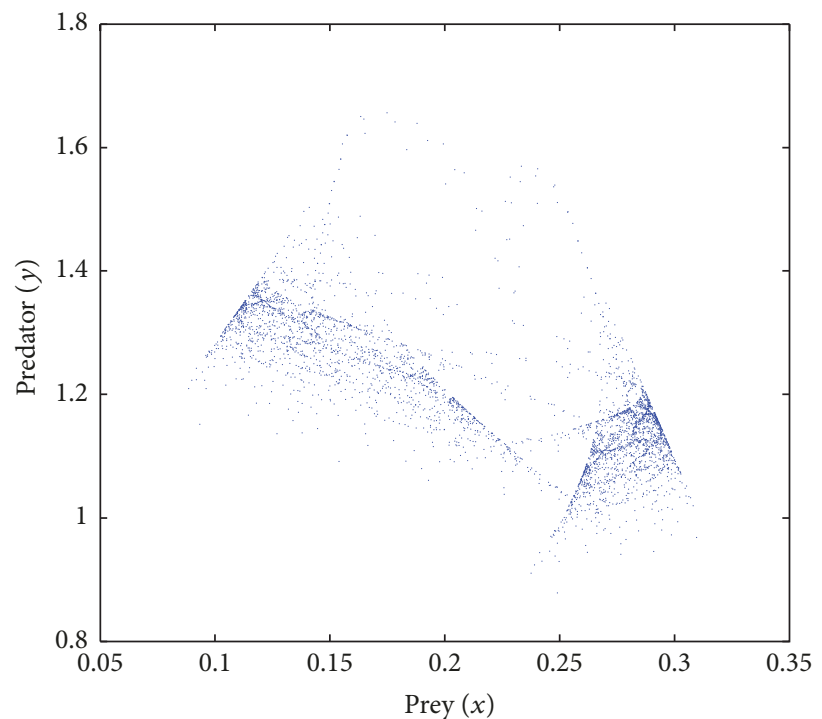

(b) $\delta=4.36$

FIgURE 4: Chaotic phase portraits of system (3) when $a=0.8, b=0.3, c=0.5, D=0.1, h=0.3, k=1$. (a) $\delta=4.33$. (b) $\delta=4.36$. 


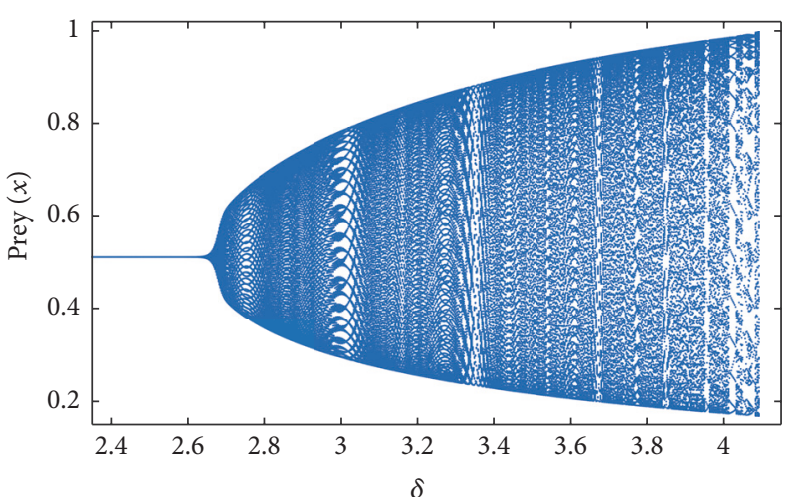

(a)

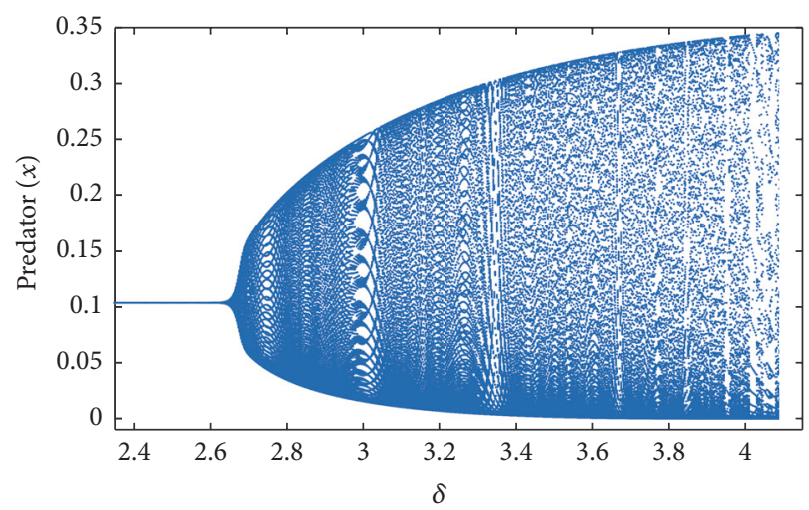

(c)

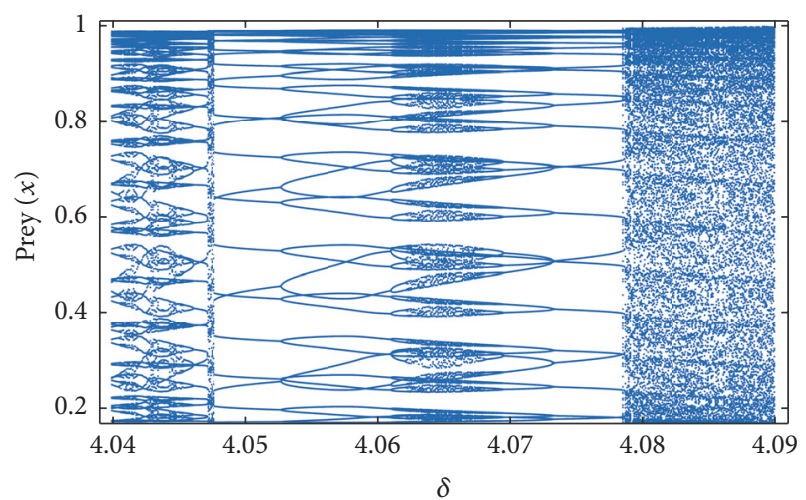

(b)

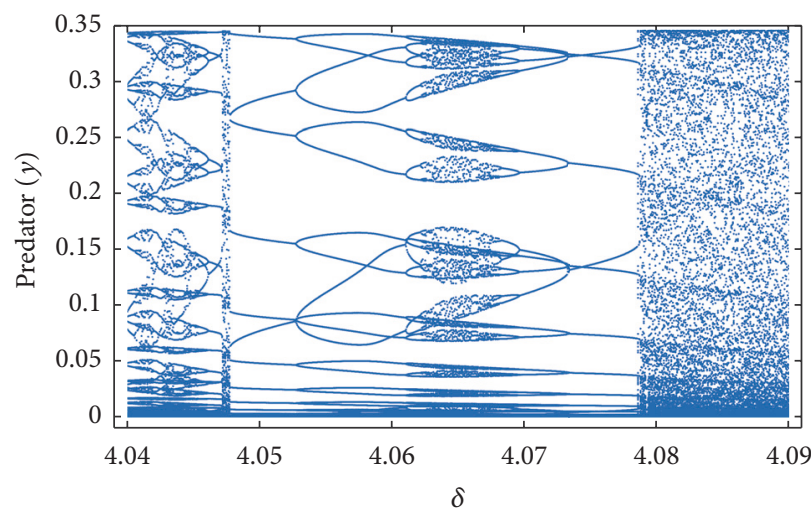

(d)

Figure 5: (a) Bifurcation diagram of system (3) in the $(\delta, x)$ plane when $a=0.1, b=1, c=1, D=0.4, h=0.6, k=1$, and $2.35 \leq \delta \leq 4.15$. The initial values is $(0.5118,0.1051)$. (b) Local amplification corresponding to (a) for $\delta \in[4.04,4.09]$. (c) Bifurcation diagram of system (3) in the $(\delta, y)$ plane. (d) Local amplification corresponding to (c) for $\delta \in[4.04,4.09]$.

the invariant circle occurs, its diameter is bigger and bigger according the value of $\delta$. In addition, period-doubling and period-halving phenomena are also observed in Figures 5(b) and 5(d) which are the local amplification of Figures 5(a) and 5(c), respectively, for $\delta \in[4.04,4.09]$. In fact, if the value of $\delta$ exceeds 4.048, as shown in Figure 8, orbits of period 25 and 50 appear. Also, periodic-halving phenomenon is detected in Figure 9. The maximum Lyapunov exponents corresponding to Figure 5(a) are calculated and plotted in Figure 6 to display the dynamical complexity of system (3).

\section{Conclusion and Discussion}

In summary, we considered the discrete-time predator-prey system with the step size $\delta$ obtained from a continuous predator-prey system with Ivlev functional response. Firstly, we found all fixed points of the system and investigated their stability via the eigenvalues of Jacobian matrix. Based on this analysis, we provided the parameter sets for which the system undergoes a flip bifurcation and Hopf bifurcation according to the value $\delta$ by using the center manifold theorem and bifurcation theory. In addition, we demonstrated numerical simulations including phase portraits and bifurcation diagrams of the system in order to validate our mathematical results and show that the system has complex dynamical behaviors such as a cascade of periodic doubling, an attracting invariant circle, or chaotic attractors. Also, we computed the Lyapunov exponents to confirm the dynamical complexity of the system.

In fact, it follows from Theorem 5 that system (3) can have a flip bifurcation, which results in periodic doubling and chaotic phenomena as shown in Figures 1 and 4. Here the chaotic phenomenon indicates biologically that the biological system (3) is not stable and, particularly, if the prey is in chaotic, then the predator will eventually go extinct or go to a stable equilibrium. Also Theorem 6 guarantees the existence of a closed curve as shown in Figure 7, which means biologically that the predator coexists with the prey with at least two-frequency oscillation.

There is an interesting thing that the parameter $h$, the maximum rate of predation, has no effect on qualitative dynamical behaviors including the stability of the fixed points and the existence of bifurcation phenomena. For instant, if we take $h=0.1$ and $h=0.2$ instead of $h=0.3$ and $h=0.6$ in cases (i) and (ii), respectively, we can obtain qualitatively similar bifurcation diagrams in Figure 10 to Figures 1 and 5 .

As mentioned in the introduction, there are many works about discrete-time predator-prey systems with Holling-type functional responses. Now we will compare the dynamics of these systems with that of system (3). If a predator-prey 


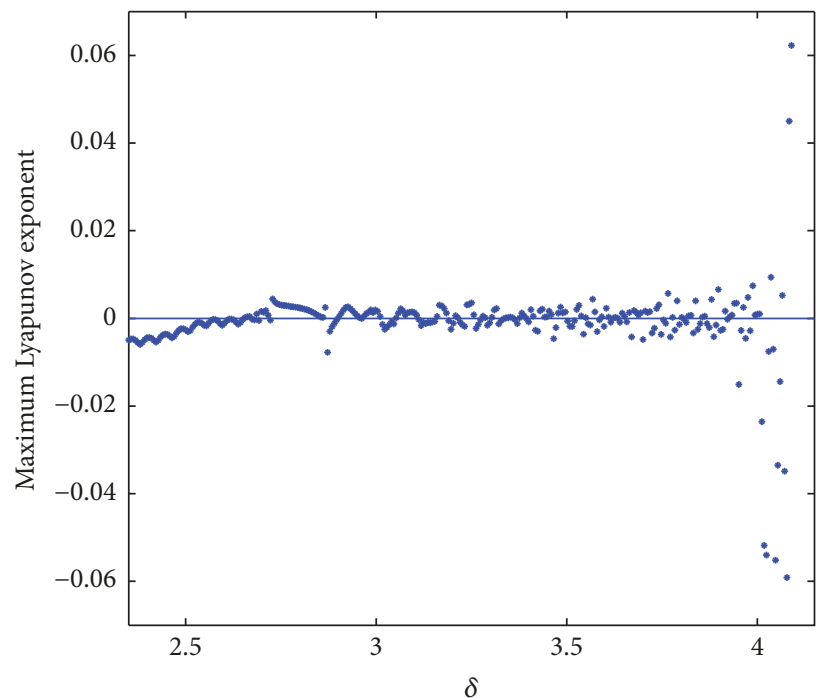

(a) $2.35 \leq \delta \leq 4.15$

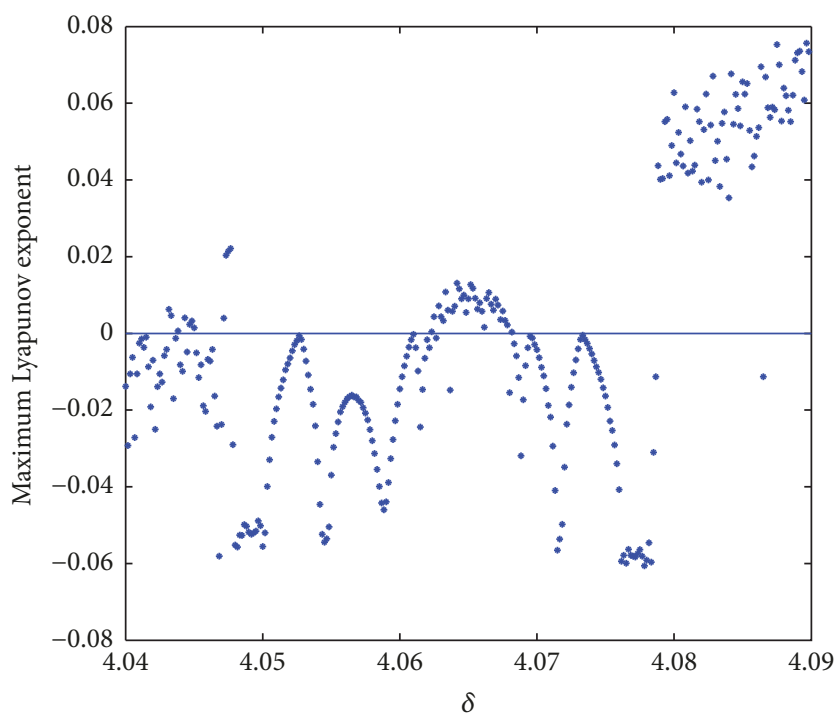

(b) $4.04 \leq \delta \leq 4.09$

Figure 6: (a) Maximum Lyapunov exponents of system (3) corresponding to Figure 5(a). (b) Local amplification corresponding to (a) for $\delta \in[4.04,4.09]$.

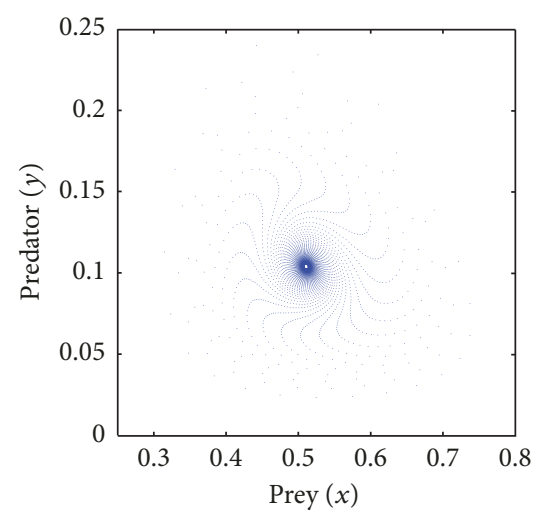

(a) $\delta=2.6$

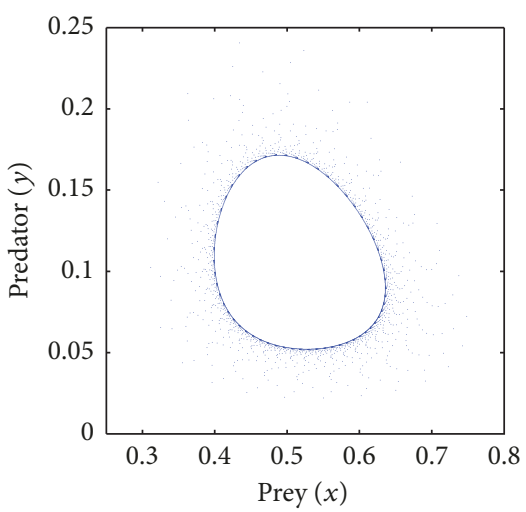

(b) $\delta=2.72$

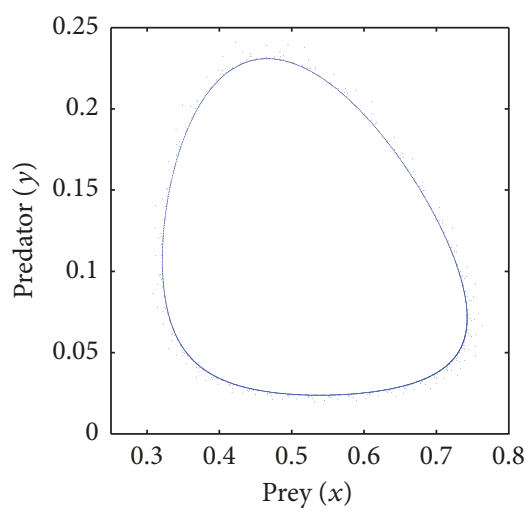

(c) $\delta=2.9$

Figure 7: (a) Phase portrait of a stable fixed point of system (3) when $a=0.1, b=1, c=1, D=0.4, h=0.6, k=1$, and $\delta=2.6$. (b) Phase portrait of system (3) after Hopf bifurcation when $\delta=2.72$. (c) Phase portrait of system (3) after Hopf bifurcation when $\delta=2.9$. Here, the initial point is $(0.6108,0.2041)$.

system has stable positive fixed points, then all species of the system could coexist, which is a biologically significant fact from the point of view of biodiversity. In this context, we will focus on positive fixed points. We summarize the dynamical behavior of systems around positive fixed points in Table 1 by letting LAS, SNB, FB, and HB stand for locally asymptotically stable, saddle-node bifurcation, flip bifurcation, and Hopf bifurcation, respectively.

Comparing the first row to the second row of Table 1, we figure out that the discrete-time predator-prey system exhibits much richer dynamics than the continuous-time system. From the second row to the fifth row, we assert that the discrete-time predator-prey systems have similar dynamical behaviors around their positive fixed points even if their functional responses are different from each other.
Moreover, we conclude that the discrete-time systems have various dynamical behaviors regardless of the functional responses.

It can be inferred from the results obtained in this paper that the Ivlev functional response may be a cause of chaoslike oscillation of populations and can make the behavior of population systems more complex. Nevertheless, identifying complicated, possibly chaotic dynamics in population data has remained a major challenge in ecological studies $[34,35]$. However, this work could be very helpful for the biologists who work with discrete-time prey-predator systems.

\section{Conflicts of Interest}

The author declares that there are no conflicts of interest. 


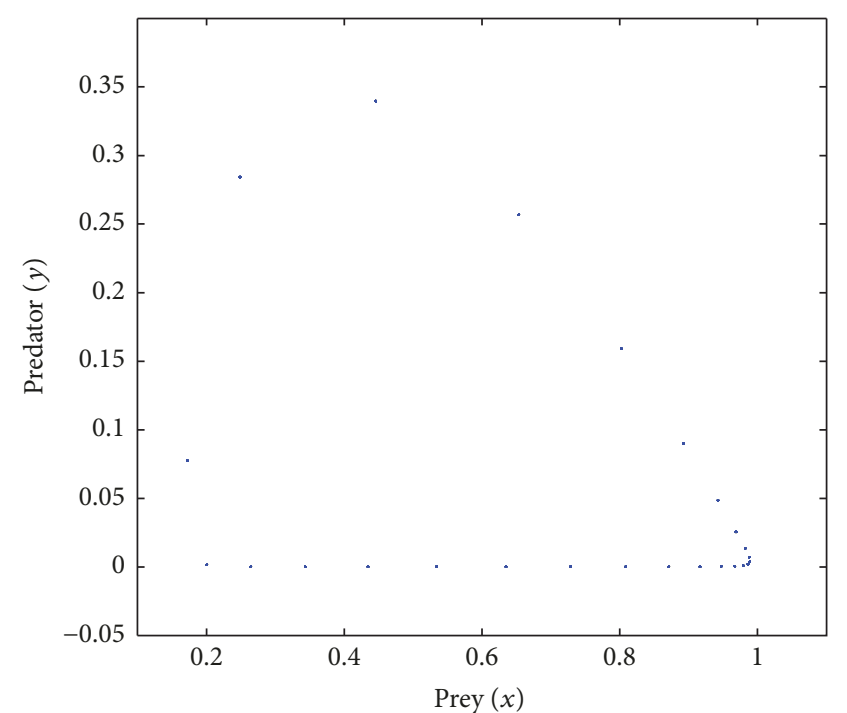

(a) $\delta=4.05$

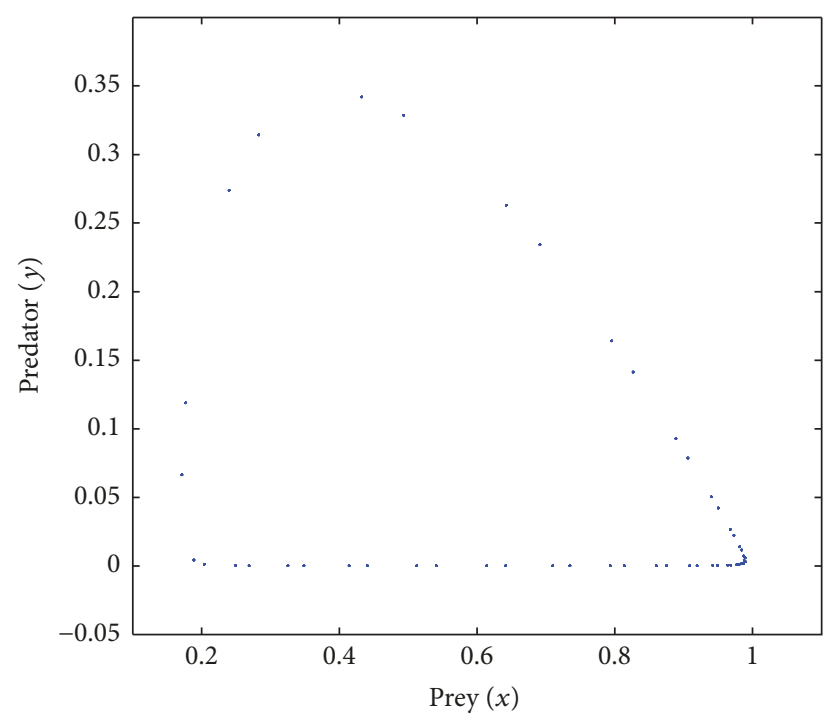

(b) $\delta=4.056$

Figure 8: (a) Phase portrait of a period-25 orbit of system (3) when $a=0.1, b=1, c=1, D=0.4, h=0.6, k=1$, and $\delta=4.05$. (b) Phase portrait of a period-50 orbit when $\delta=4.056$.

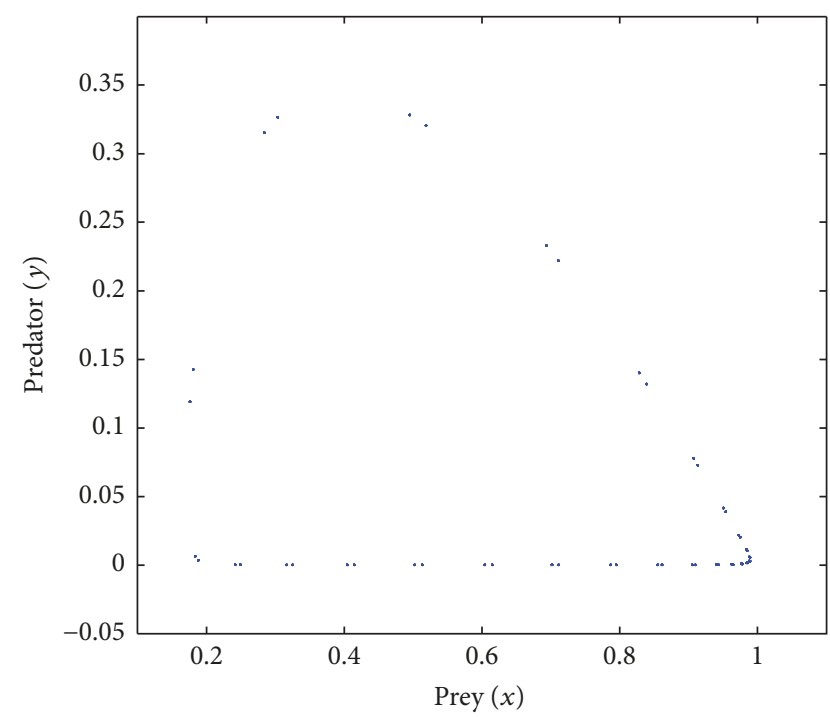

(a) $\delta=4.072$

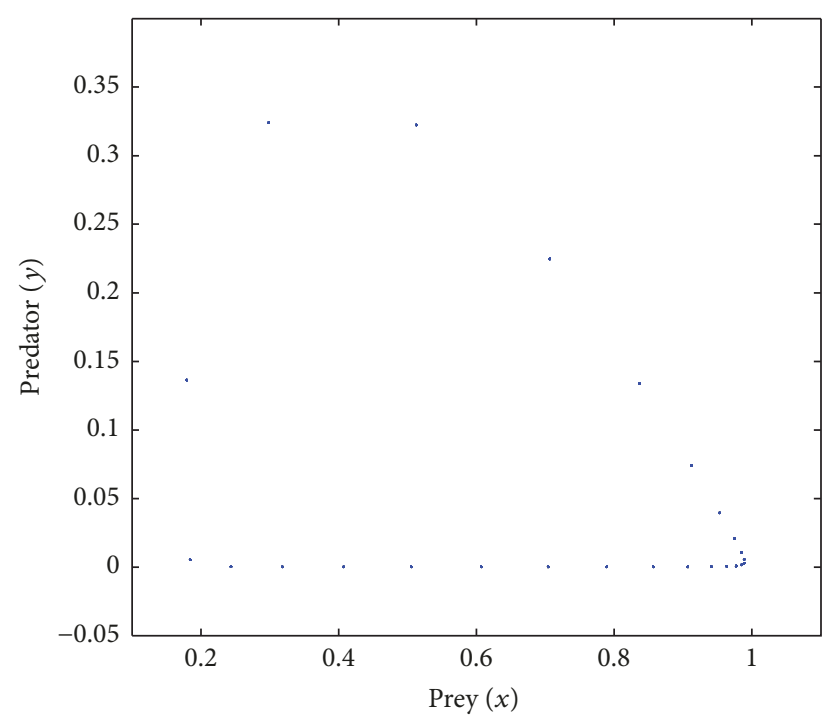

(b) $\delta=4.075$

Figure 9: (a) Phase portrait of a period-50 orbit of system (3) when $a=0.1, b=1, c=1, D=0.4, h=0.6, k=1$, and $\delta=4.072$. (b) Phase portrait of a period- 25 orbit when $\delta=4.075$.

TABLE 1: This table lists the stability and bifurcations of systems according to functional response.

\begin{tabular}{lcccccc}
\hline Systems & Existence & LAS & SNB & FB & HB & References \\
\hline System (2) & Yes & Yes & No & No & No & {$[23]$} \\
System (3) & Yes & Yes & No & Yes & Yes & - \\
Lotka-Volterra & Yes & Yes & No & Yes & Yes & {$[15]$} \\
Holling II & Yes & Yes & No & Yes & Yes & {$[29]$} \\
Holling III & Yes & Yes & No & Yes & Yes & {$[13]$} \\
Holling IV & Yes & Yes & Yes & Yes & Yes & {$[31]$} \\
\hline
\end{tabular}




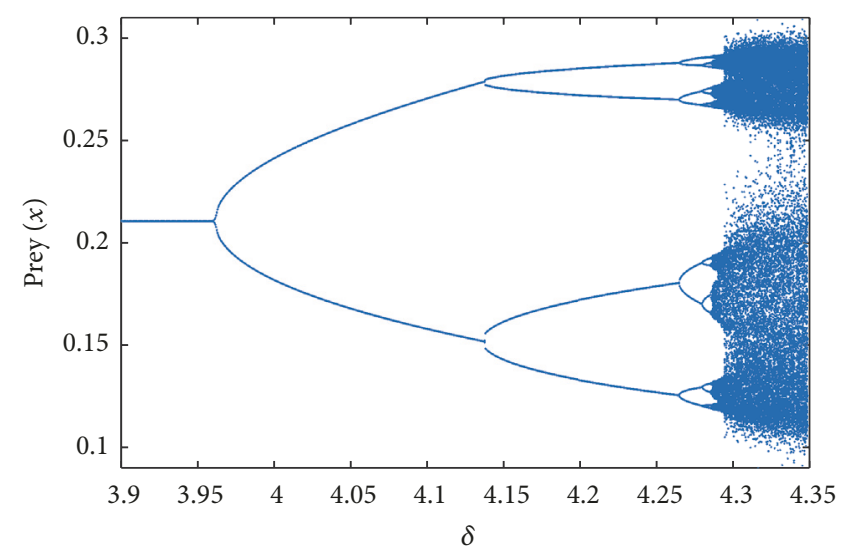

(a)

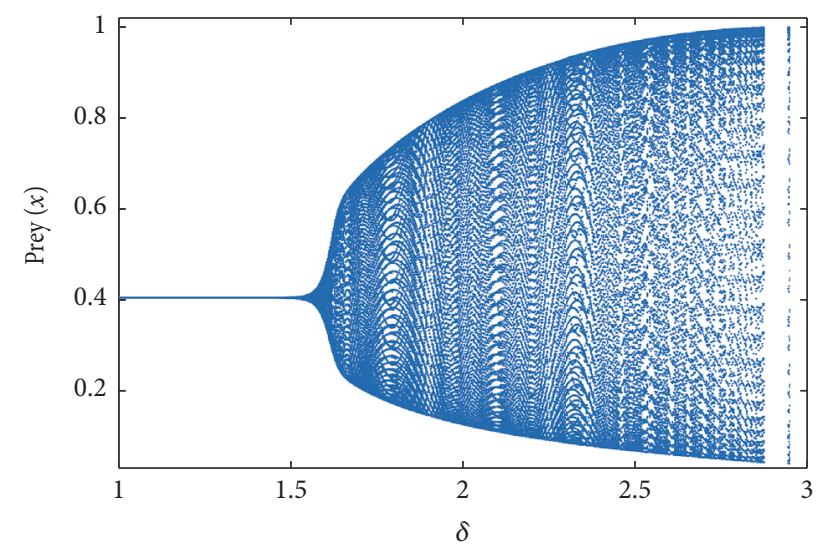

(c)

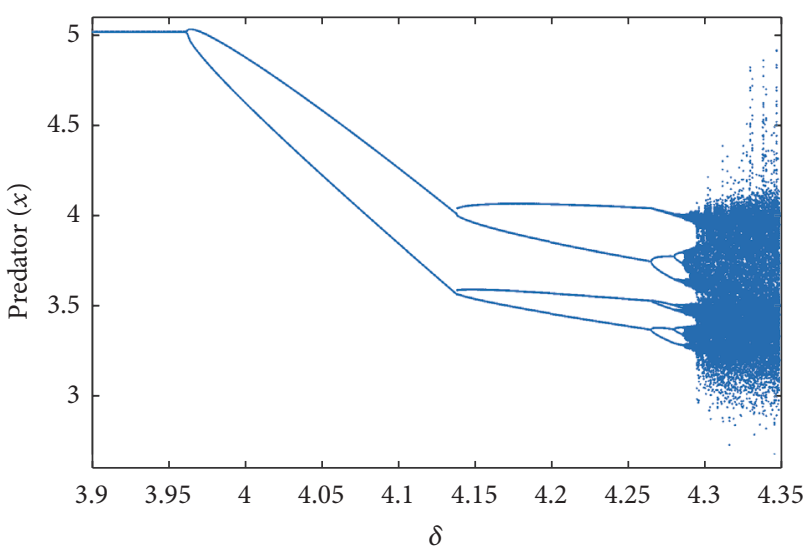

(b)

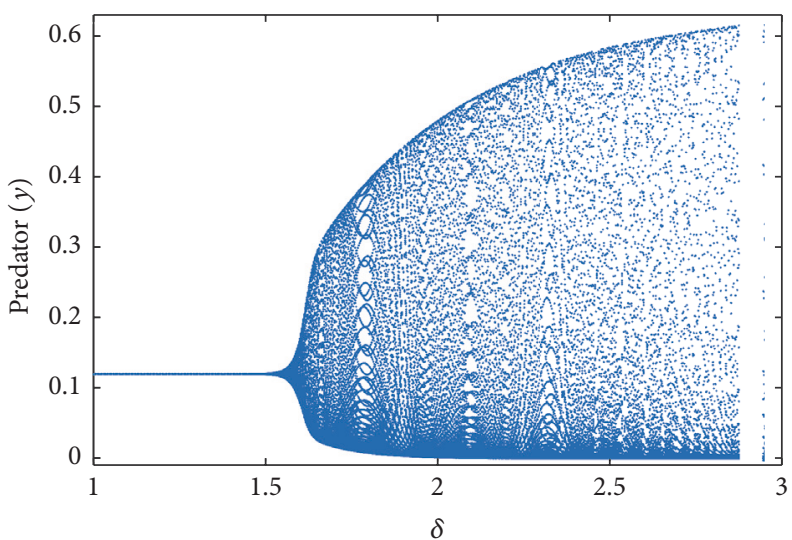

(d)

Figure 10: (a) Bifurcation diagram of system (3) in the $(\delta, x)$ plane when $a=0.1, b=1, c=1, D=0.4, h=0.1, k=1$, and 3.9 $\leq \delta \leq 4.35$. (b) Bifurcation diagram of system (3) in the $(\delta, y)$. (c) Bifurcation diagram of system (3) in the $(\delta, x)$ plane when $a=0.1, b=1, c=1, D=0.4$, $h=0.2, k=1$, and $1 \leq \delta \leq 3$. (d) Bifurcation diagram of system (3) in the $(\delta, y)$.

\section{Acknowledgments}

This work was supported by Basic Science Research Program through the National Research Foundation of Korea (NRF) funded by the Ministry of Education (2016R1D1A3B01009330).

\section{References}

[1] R. Arditi and L. R. Ginzburg, "Coupling in predator-prey dynamics: ratio-dependence," Journal of Theoretical Biology, vol. 139, no. 3, pp. 311-326, 1989.

[2] C. Cosner, D. L. Deangelis, J. S. Ault, and D. B. Olson, "Effects of spatial grouping on the functional response of predators," Theoretical Population Biology, vol. 56, no. 1, pp. 65-75, 1999.

[3] M. Danca, S. Codreanu, and B. Bakó, "Detailed analysis of a nonlinear prey-predator model," Journal of Biological Physics, vol. 23, no. 1, pp. 11-20, 1997.

[4] M. Fan and Y. Kuang, "Dynamics of a nonautonomous predator-prey system with the Beddington-DeAngelis functional response," Journal of Mathematical Analysis and Applications, vol. 295, no. 1, pp. 15-39, 2004.

[5] B. Liu, Y. Zhang, and L. Chen, "Dynamic complexities in a Lotka-Volterra predator-prey model concerning impulsive control strategy," International Journal of Bifurcation and Chaos, vol. 15, no. 2, pp. 517-531, 2005.

[6] S. Ruan and D. Xiao, "Global analysis in a predator-prey system with nonmonotonic functional response," SIAM Journal on Applied Mathematics, vol. 61, no. 4, pp. 1445-1472, 2000.

[7] E. Saez and E. Gonzalez-Olivares, "Dynamics of a predator-prey model," SIAM Journal on Applied Mathematics, vol. 59, no. 5, pp. 1867-1878, 1999.

[8] W. Wang, Q.-X. Liu, and Z. Jin, "Spatiotemporal complexity of a ratio-dependent predator-prey system," Physical Review E: Statistical, Nonlinear, and Soft Matter Physics, vol. 75, no. 5, Article ID 051913, 2007.

[9] H. N. Agiza, E. M. ELabbasy, H. EL-Metwally, and A. A. Elsadany, "Chaotic dynamics of a discrete prey-predator model with Holling type II," Nonlinear Analysis: Real World Applications, vol. 10, no. 1, pp. 116-129, 2009.

[10] Q. Din, "Controlling chaos in a discrete-time prey-predator model with Allee effects," International Journal of Dynamics and Control, 2017.

[11] Q. Din, "Complexity and chaos control in a discrete-time preypredator model," Communications in Nonlinear Science and Numerical Simulation, vol. 49, pp. 113-134, 2017.

[12] Z. He and X. Lai, "Bifurcation and chaotic behavior of a discretetime predator-prey system," Nonlinear Analysis: Real World Applications, vol. 12, no. 1, pp. 403-417, 2011. 
[13] Z. He and B. Li, "Complex dynamic behavior of a discretetime predator-prey system of Holling-III type," Advances in Difference Equations, vol. 2014, no. 1, p. 180, 2014.

[14] Z. Hu, Z. Teng, and L. Zhang, "Stability and bifurcation analysis of a discrete predator-prey model with nonmonotonic functional response," Nonlinear Analysis: Real World Applications, vol. 12, no. 4, pp. 2356-2377, 2011.

[15] X. Liu and D. Xiao, "Complex dynamic behaviors of a discretetime predator-prey system," Chaos, Solitons and Fractals, vol. 32, no. 1, pp. 80-94, 2007.

[16] X. Liu, Y. Liu, and Q. Li, "Multiple bifurcations and chaos in a discrete prey-predator system with generalized holling III functional response," Discrete Dynamics in Nature and Society, vol. 2015, Article ID 245421, 10 pages, 2015.

[17] C. S. Holling, "The functional response of predators to prey density and its role in mimicry and population regulation," Memoirs of the Entomological Society of Canada, vol. 97, supplement 45, pp. 5-60, 1965.

[18] J. F. Andrews, "A mathematical model for the continuous culture of microorganisms utilizing inhibitory substrates," Biotechnology and Bioengineering, vol. 10, no. 6, pp. 707-723, 1968.

[19] W. Sokol and J. Howell, "Kinetics of phenol oxidation by washed cells," Biotechnology and Bioengineering, vol. 23, no. 9, pp. 20392049, 1980.

[20] V. S. Ivlev, Experimental Ecology of The Feeding of Fishes, Yale University Press, Kentucky, Ky, USA, 1961.

[21] K. S. Cheng, S. B. Hsu, and S. S. Lin, "Some results on global stability of a predator-prey system," Journal of Mathematical Biology, vol. 12, no. 1, pp. 115-126, 1981.

[22] G. Guo, B. Li, and X. Lin, "Qualitative analysis on a predatorprey model with Ivlev functional response," Advances in Difference Equations, vol. 2013, article 164, 2013.

[23] R. E. Kooij, "A predator-prey model with Ivlev's functional response," Journal of Mathematical Analysis and Applications, vol. 198, no. 2, pp. 473-489, 1996.

[24] R. M. May, "Limit cycles in predator-prey communities," Science, vol. 177, no. 4052, pp. 900-902, 1972.

[25] W. Wang, L. Zhang, H. Wang, and Z. Li, "Pattern formation of a predator-prey system with Ivlev-type functional response," Ecological Modelling, vol. 221, no. 2, pp. 131-140, 2010.

[26] I. G. Pearce, M. A. Chaplain, P. . Schofield, A. R. Anderson, and S. F. Hubbard, "Modelling the spatio-temporal dynamics of multi-species host-parasitoid interactions: heterogeneous patterns and ecological implications," Journal of Theoretical Biology, vol. 241, no. 4, pp. 876-886, 2006.

[27] K. F. Preedy, P. G. Schofield, M. A. J. Chaplain, and S. F. Hubbard, "Disease induced dynamics in host-parasitoid systems: chaos and coexistence," Journal of the Royal Society Interface, vol. 4, no. 14, pp. 463-471, 2007.

[28] K. Uriu and Y. Iwasa, "Turing pattern formation with two kinds of cells and a diffusive chemical," Bulletin of Mathematical Biology, vol. 69, no. 8, pp. 2515-2536, 2007.

[29] Z. Jing and J. Yang, "Bifurcation and chaos in discrete-time predator-prey system," Chaos, Solition and Fractals, vol. 27, pp. 259-277, 2006.

[30] X. Liu and Y. Xing, "Bifurcations of a ratio-dependent Hollingtanner system with refuge and constant Harvesting," Abstract and Applied Analysis, vol. 2013, Article ID 478315, 10 pages, 2013.

[31] Q. Chen, Z. Teng, and Z. Hu, "Bifurcation and control for a discrete-time prey-predator model with Holling-IV functional response," International Journal of Applied Mathematics and Computer Science, vol. 23, no. 2, pp. 247-261, 2013.
[32] C. Robinson, Dynamical Systems, Stability, Symbolic Dynamics and Chaos, CRC Press, Boca Raton, Fla, USA, 2nd edition, 1999.

[33] J. Guckenheimer and P. Holmes, Nonlinear oscillations, dynamical system and bifurcation of vector fields, Springer, New York, NY, USA, 1983.

[34] G. Sugihara and R. M. May, "Nonlinear forecasting as a way of distinguishing chaos from measurement error in time series," Nature, vol. 344, no. 6268, pp. 734-741, 1990.

[35] Y. Xiao, D. Cheng, and S. Tang, "Dynamic complexities in predator-prey ecosystem models with age-structure for predator," Chaos, Solitons and Fractals, vol. 14, no. 9, pp. 1403-1411, 2002 . 


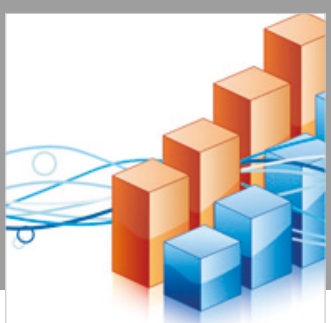

Advances in

Operations Research

\section{-n-m}
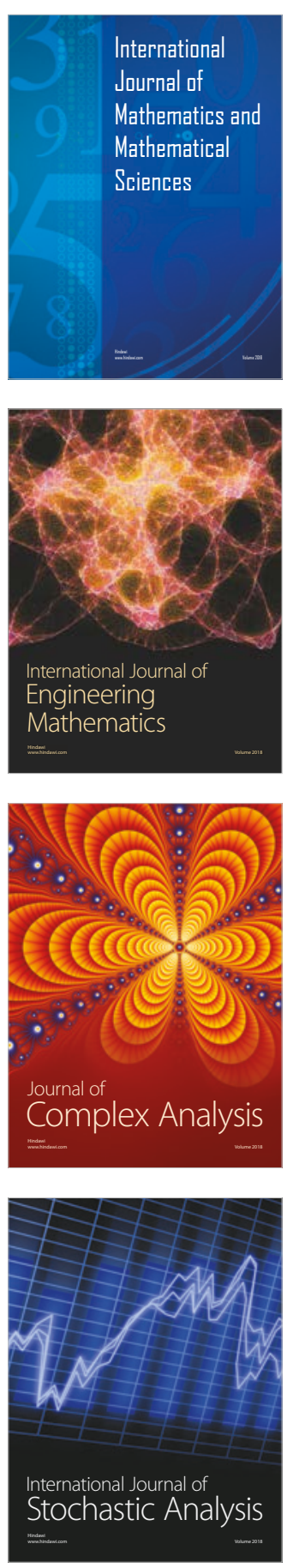
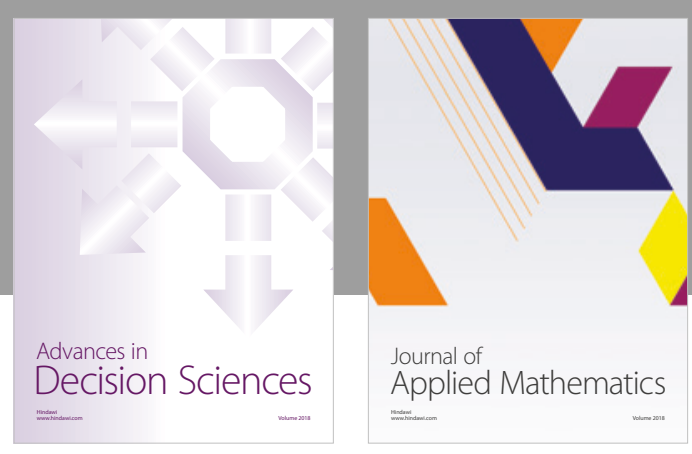

Journal of

Applied Mathematics
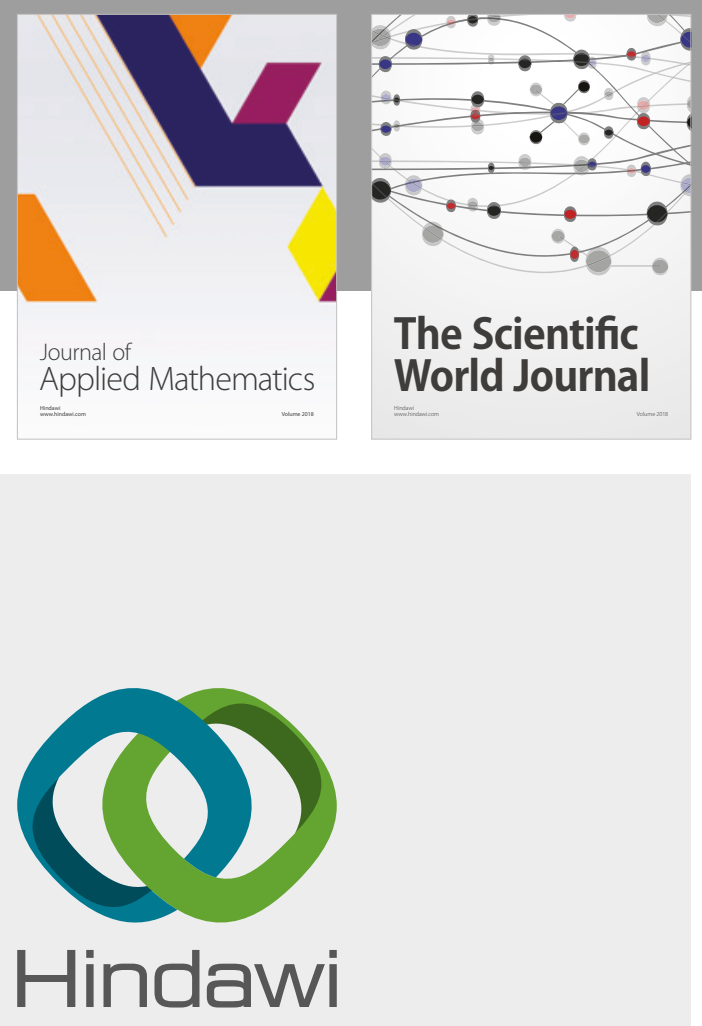

Submit your manuscripts at

www.hindawi.com

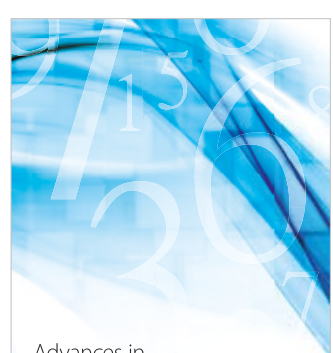

Advances in
Numerical Analysis
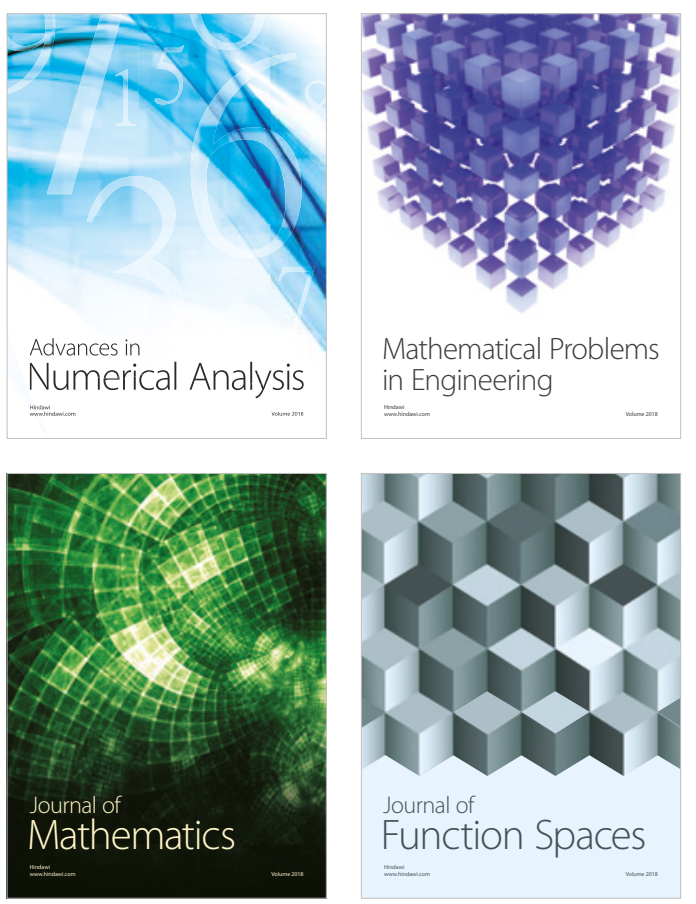

Mathematical Problems in Engineering

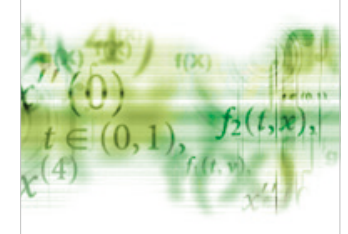

International Journal of

Differential Equations

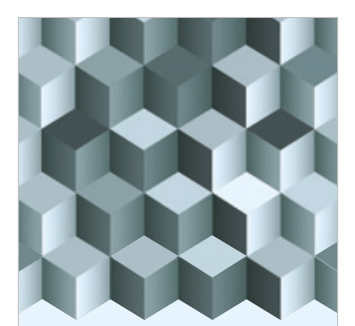

Journal of

Function Spaces

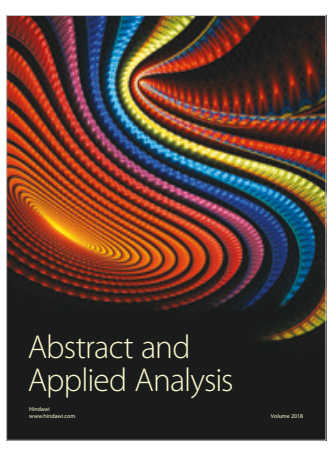

The Scientific

World Journal

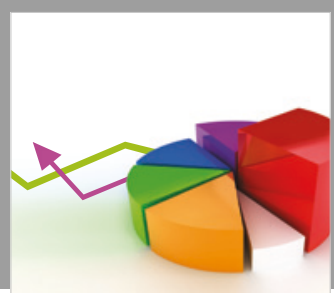

Journal of

Probability and Statistics
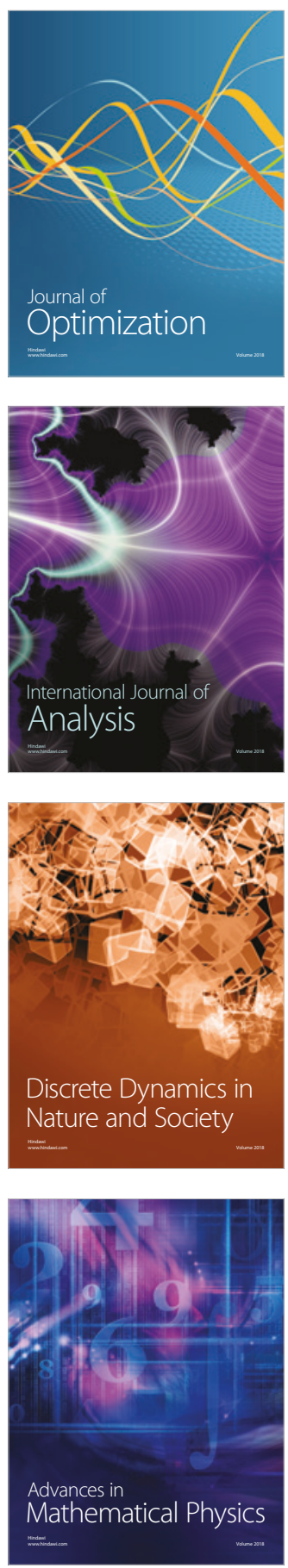